Revue des patrimoines

Le patrimoine militaire et la question urbaine

\title{
De la Seine au plateau : l'impact de la présence militaire sur l'urbanisme de Melun
}

Judith Förstel

\section{(2) OpenEdition}

Journals

Édition électronique

URL : http://journals.openedition.org/insitu/141

DOI : 10.4000/insitu. 141

ISSN : 1630-7305

Éditeur

Ministère de la culture

Référence électronique

Judith Förstel, « De la Seine au plateau : l'impact de la présence militaire sur l'urbanisme de Melun », In Situ [En ligne], 16 | 2011, mis en ligne le 27 juin 2011, consulté le 20 avril 2019. URL : http:// journals.openedition.org/insitu/141; DOI : 10.4000/insitu.141

Ce document a été généré automatiquement le 20 avril 2019.

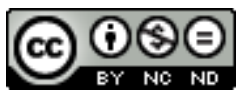

In Situ Revues des patrimoines est mis à disposition selon les termes de la licence Creative Commons Attribution - Pas d'Utilisation Commerciale - Pas de Modification 4.0 International. 


\title{
De la Seine au plateau : l'impact de la présence militaire sur l'urbanisme de Melun
}

\author{
Judith Förstel
}

1 La présence de l'armée à Melun, préfecture du département de Seine-et-Marne située à une cinquantaine de kilomètres au sud-est de Paris, a eu une incidence non négligeable sur le développement de la ville au $\mathrm{XIX}^{\mathrm{e}}$ et au $\mathrm{XX}^{\mathrm{e}}$ siècle. Même si Melun n'a pas particulièrement l'image d'une ville de garnison, elle a pourtant accueilli tout au long de ces deux siècles des soldats en nombre assez important, eu égard à la taille assez modeste de l'agglomération. 1300 hommes (et 718 chevaux) y sont ainsi cantonnés en 1852, pour une population totale de 7750 âmes. Cet effectif est comparable à celui d'autres villes mêlant activités administratives et présence militaire, à l'instar de Laon qui comptait, à la même époque, une caserne de 800 hommes dans la citadelle et un quartier de cavalerie ayant une capacité de 644 hommes - à cette différence près que Laon est restée une «place de guerre» jusqu'au déclassement du site en 1851, ce qui a impliqué la conservation et l'entretien des fortifications ${ }^{1}$. Le nombre de soldats cantonnés à Melun pendant tout le XIX siècle a par ailleurs augmenté régulièrement, quoique sans atteindre le rythme d'accroissement effréné qu'a pu connaître une ville comme Châlons-enChampagne (6000 hommes à la veille de la Première Guerre mondiale !) ${ }^{2}$. Sans prétendre à une quelconque spécificité, le cas melunais, étudié dans le cadre de l'Inventaire topographique ${ }^{3}$, pourra donc permettre de saisir l'importance des rapports entre les autorités municipales et militaires dans l'évolution de la trame urbaine. 
Figure 1

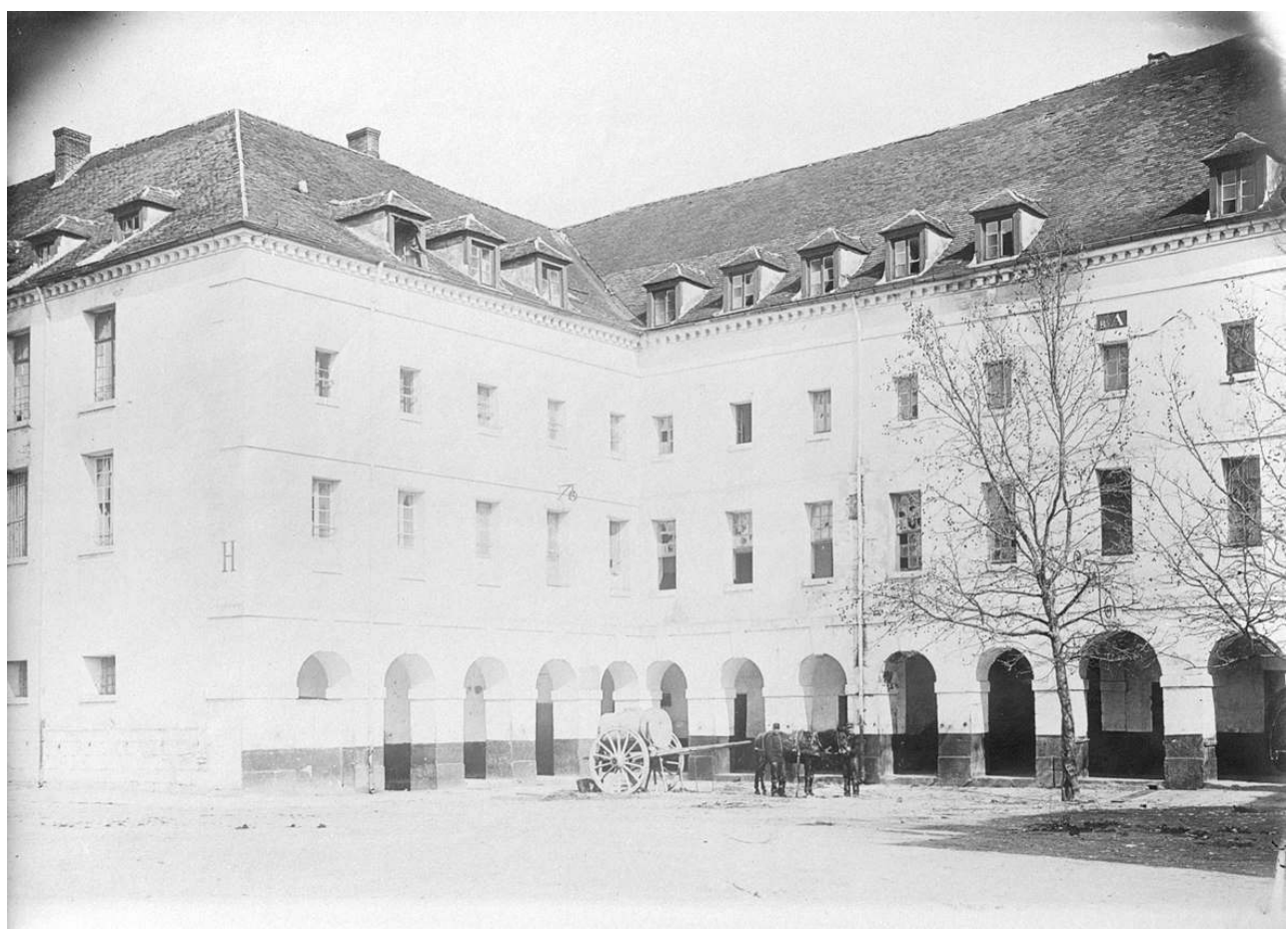

Le quartier de cavalerie Augereau, principal site de casernement à Melun au XIX'e siècle. Photographie du corps de logis nord, vers 1900. Collection particulière. Repro. Asseline, Stéphane.

(c) Conseil régional d'île-de-France, service Patrimoines et Inventaire, 2004.

On peut distinguer, dans cette évolution, deux périodes distinctes liées à l'extension puis à l'abandon du principal site de cantonnement des soldats, sur la rive sud de la Seine (fig. $\mathbf{n}^{\circ}$ 1). En 1780, Melun s'est en effet dotée d'un quartier de cavalerie installé dans un ancien couvent, en bordure du fleuve. Cet établissement a connu un développement ininterrompu jusqu'à la fin du XIX siècle, couvrant alors une superficie de plus de six hectares ; toute une partie de la ville fut ainsi « gelée » par l'occupation militaire. Mais en 1905, cette caserne est transférée sur le plateau nord. L'espace ainsi dégagé fut l'occasion pour la municipalité d'engager un important programme d'aménagement du site, que l'arrivée du chemin de fer au milieu du XIX ${ }^{e}$ siècle avait rendu très attractif. En même temps, l'installation d'un vaste quartier militaire flambant neuf au nord de la ville entraîna également l'urbanisation de nouveaux secteurs, le long du boulevard de la République percé pour desservir les nouvelles casernes sans encombrer le centre-ville.

3 Sans entrer dans le détail de la construction des divers bâtiments militaires, par ailleurs bien documentée grâce aux archives du Génie ${ }^{4}$, nous nous proposons donc de suivre les conséquences urbanistiques de ces différentes implantations, qui ont eu un fort impact sur la croissance urbaine (fig. $\mathbf{n}^{\circ} 2$ ). 
Figure 2

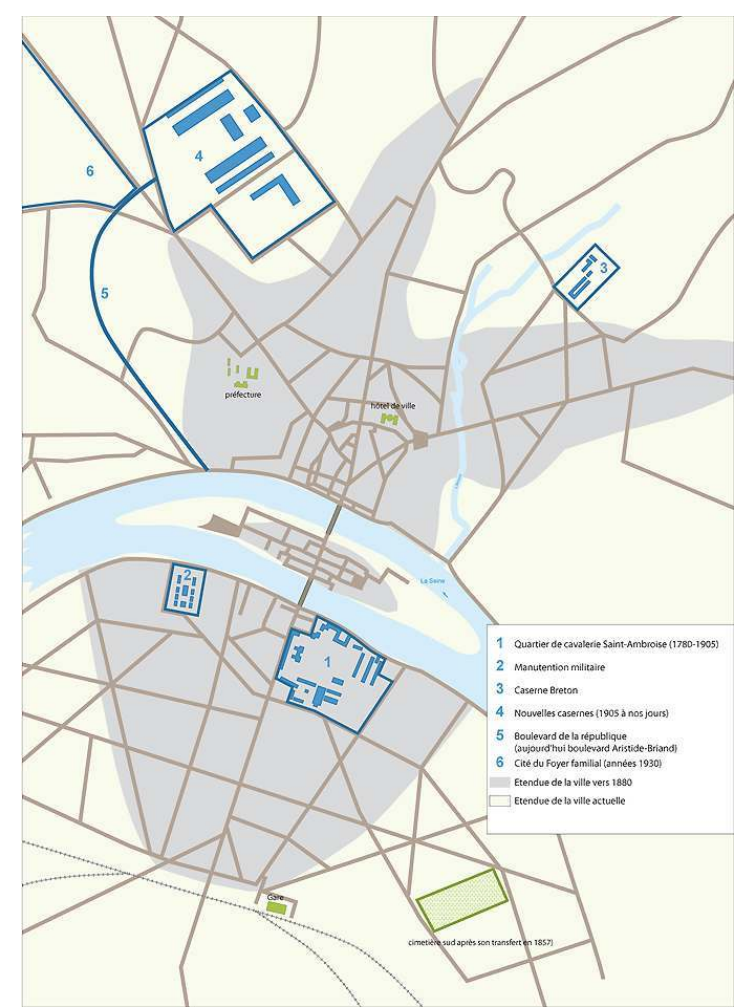

Plan de localisation des diverses implantations militaires à Melun auX XIXe-XXe siècles. Bétored, Diane. (c) Conseil régional d'Île-de-France, service Patrimoines et Inventaire, 2010.

\section{Une présence militaire en continuelle expansion (1780-1900)}

4 Tout au long de son histoire, Melun - qui verrouille l'accès à Paris en amont de la Seine a régulièrement abrité des troupes. Au Moyen Âge, elles occupaient le château royal (aujourd'hui disparu) à la pointe occidentale de l'île Saint-Étienne. À partir du XVI ${ }^{\mathrm{e}}$ siècle, le roi préféra résider à Fontainebleau lorsqu'il chassait en forêt de Bière ; mais Melun n'en continua pas moins à accueillir un régiment des Gardes françaises. Ces troupes étaient logées chez l'habitant, avec les nuisances que cela implique. D'où le projet de construire une caserne pour abriter ces soldats, selon la politique alors mise en œuvre par le Régent dans l'ensemble du royaume (et non plus seulement aux frontières). En application de l'ordonnance du 26 septembre 1719, l'ingénieur militaire Antoine Mazin avait proposé à cet effet un plan-type de casernes dont la construction se voulait économique ; on n'en prévoyait pas moins de 496 - chiffre qui ne fut bien sûr jamais atteint ${ }^{5}$. Dès 1719, l'intendant de la généralité de Paris fixa l'emplacement de la future caserne de Melun sur le site de l'ancien château royal, qui était alors en grande partie ruiné (il fut à plusieurs reprises utilisé comme carrière de pierres, à partir de 1689). Mazin dressa le plan de situation de ces casernes en 1724 (fig. $\mathbf{n}^{\circ}$ 3). On commença à démolir les vestiges du château jugés inutiles en recourant à la corvée, et dès 1724 les deux tours flanquant la porte d'entrée étaient abattues ${ }^{6}$. Mais en 1737 , l'État reporta la charge de ce projet sur la Ville: l'ancien château fut cédé à la municipalité moyennant dix livres de rente, à 
condition « d'y faire construire des cazernes conformément aux plan et devis $»^{7}$. En fait, la Ville se montra incapable de faire aboutir le chantier et finit par louer les vestiges du château aux fermiers des coches d'eau. Melun n'est d'ailleurs que l'un des multiples chantiers avortés de l'ordonnance de 1719. Il fallut, en définitive, attendre le dernier quart du XVIII ${ }^{e}$ siècle pour qu'une caserne soit enfin créée dans les locaux de l'ancien couvent des Visitandines, sur la rive sud de la Seine.

Figure 3

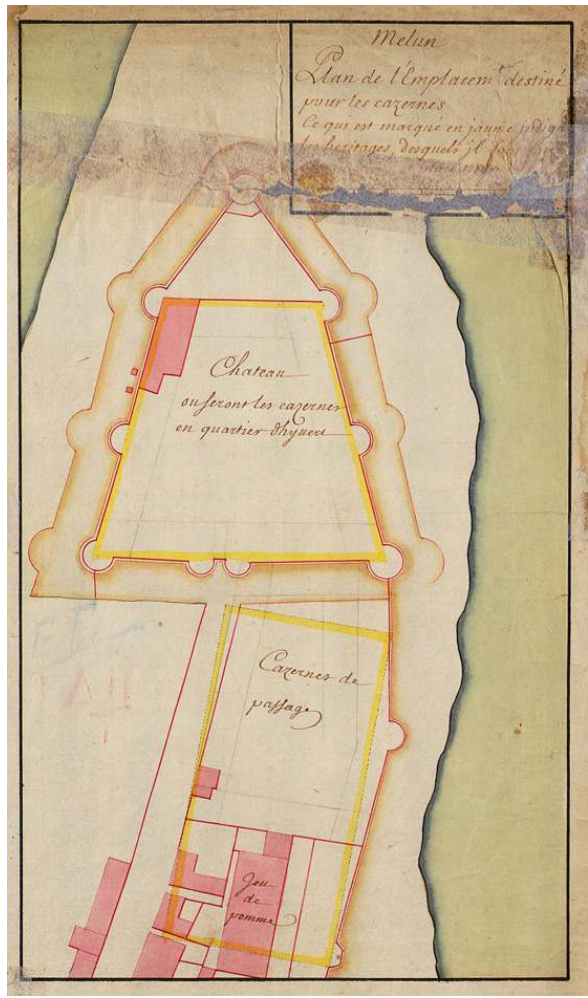

Emplacement des casernes de Melun. Plan de Marzin, 1724. Archives municipales de Melun, 1 Fi 2519. Repro. Archives de Melun.

(c) Ville de Melun/Conseil régional d'île-de-France, service Patrimoines et Inventaire, 2004.

Les Visitandines, arrivées à Melun en 1635, avaient construit un couvent qui s'était étendu de part et d'autres des fortifications médiévales, dont le roi leur avait concédé la jouissance en 1689 (fig. $\mathbf{n}^{\circ}$ 4). Cette maison ayant été fermée par lettres patentes d'août 1768, le bâtiment fut transformé en dépôt de mendicité en 1770. Cette réaffectation entraîna la construction d'un nouveau bâtiment en 1777, en adjonction à l'édifice de l'ancien couvent dont les vastes jardins offraient d'abondantes possibilités d'extension. Le dépôt de mendicité fut à son tour supprimé en mars 1779 pour établir un quartier de cavalerie; deux escadrons du régiment de La Rochefoucault s'y installèrent en juillet 1780, amorce d'une présence continue de la cavalerie dans la ville jusqu'en 1939. 


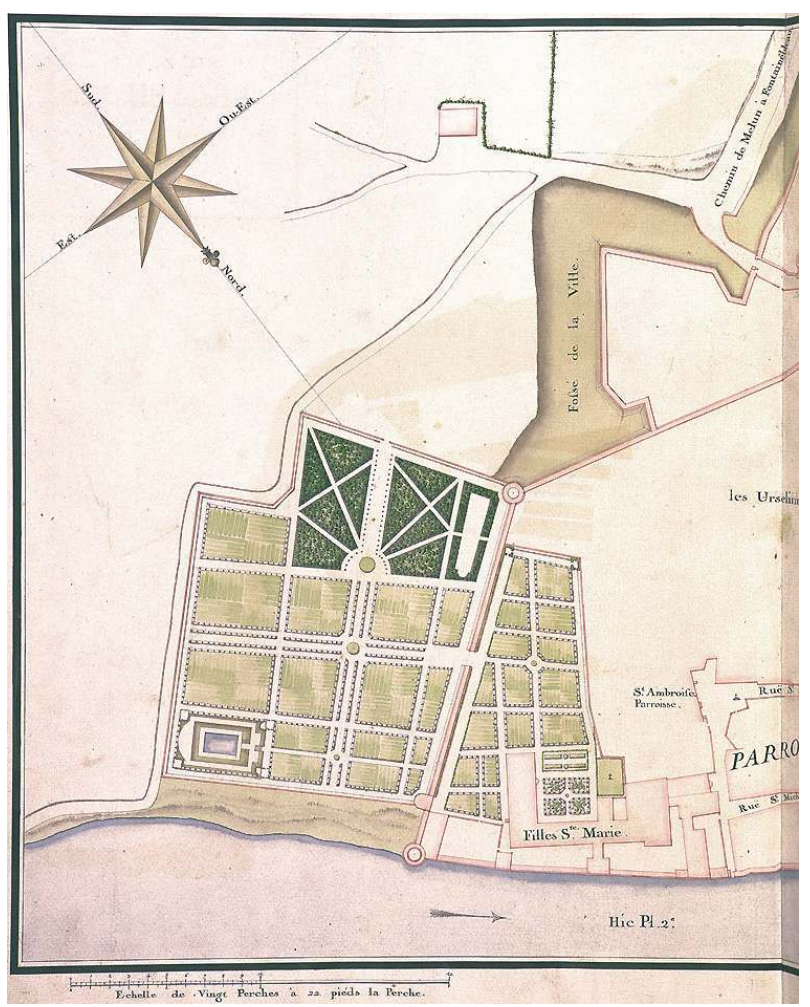

L'emprise du couvent des Visitandines en 1740. Détail du plan terrier de la vicomté de Melun, levé par P. Desquinemare. Médiathèque de Melun. Repro. Asseline, Stéphane.

(c) Ville de Melun/Conseil régional d'île-de-France, service Patrimoines et Inventaire, 2002.

Par cette implantation, Melun anticipait le grand mouvement de réaffectation d'anciens bâtiments monastiques à l'armée, qui dans la plupart des villes ne put se faire qu'à l'époque révolutionnaire, voire impériale (1801 pour la caserne Saint-Pierre à Châlons-enChampagne, par exemple). La récupération d'un édifice déjà existant permit d'autre part à la caserne de se situer en pleine ville et non à sa périphérie. Melun se démarquait ainsi des établissements militaires créés au XVIII ${ }^{\mathrm{e}}$ siècle en lisière des agglomérations, à la fois pour des raisons d'économie - les quartiers de cavalerie, avec leurs écuries et leur manège, sont grands consommateurs d'espace -, mais aussi par souci pratique : la caserne n'est pas censée être un lieu intégré à la vie urbaine. Le respect de la discipline militaire implique la clôture des lieux, et la présence des chevaux entraîne en outre des nuisances susceptibles de gêner les habitants. Les sites choisis par l'armée étaient donc généralement hors de la ville, dans des secteurs écartés de l'urbanisation : à Saumur par exemple, les Carabiniers s'installèrent sur une zone jusqu'ici inondable, auparavant occupée par une promenade, et située à l'écart des principales entrées de la ville ${ }^{8}$. À Melun en revanche, les escadrons, même s'ils ne bénéficiaient pas d'un emplacement très central (le cœur de la ville se situait plutôt sur la rive droite de la Seine, dans la paroisse Saint-Aspais), se trouvaient malgré tout intra muros, parmi les habitations particulières. Autre incidence de cette installation dans un édifice préexistant: on ne ressentit pas le besoin d'ouvrir une rue nouvelle pour desservir le quartier de cavalerie. Là encore, Melun s'écartait du modèle de la caserne construite ex nihilo, dont la création s'accompagnait volontiers d'une intervention sur le réseau viaire. À Fontenay-le-Comte, par exemple, le tracé ancien fut doublé par l'aménagement d'un nouvel axe, la rue Royale, parallèle à la 
rue médiévale (qu'il détrôna) ${ }^{9}$. Rien de tel pour le bernard-l'ermite melunais, qui dut se contenter de l'accès fort mal dégagé de l'ancien établissement monastique, par la petite rue Saint-Michel (fig. $\mathbf{n}^{\circ}$ 5).

Figure 5

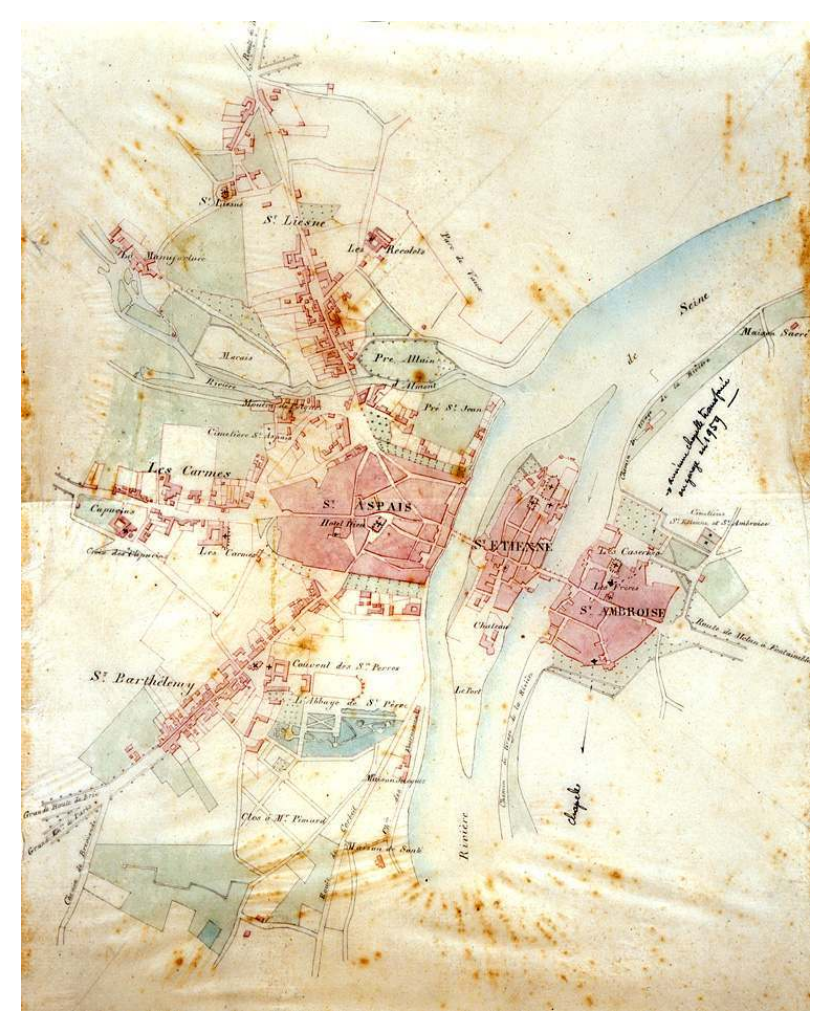

Plan de Melun en 1787, dit « plan d'intendance » (copie du XIXe siècle) ; dans la partie droite, correspondant à la paroisse Saint-Ambroise (l'église paroissiale est marquée d'une croix), « Les Casernes » occupent un espace en retrait de l'axe nord-sud qui traverse toute la ville. Archives municipales de Melun, 1 Fi 317. Repro. Asseline, Stéphane.

(c) Ville de Melun/Conseil régional d'Île-de-France, service Patrimoines et Inventaire, 2006.

7 La cavalerie ayant déjà eu droit à son couvent, la redistribution des biens du clergé menée à la Révolution s'effectua davantage en faveur des administrations civiles - à commencer par le Directoire du département nouvellement créé - que de l'armée. Celle-ci obtint néanmoins en 1800 la jouissance d'un nouveau bâtiment situé dans une partie du cidevant couvent des Carmes, au nord de la ville. Transformé en «pavillon du quartier général », il servait notamment de logement à l'État-major. Quant à la caserne installée en 1780 dans l'ancien couvent des Visitandines, sa présence ne fut pas remise en question. Toutefois, elle partageait le bâtiment avec le tribunal et la prison, dans des conditions d'ailleurs particulièrement déplorables si l'on en croit le rapport de l'architecte Peyre en l'an $\mathrm{III}^{10}$; mais ces deux institutions finirent par gagner un autre emplacement, celui du couvent des Frères de la Doctrine chrétienne (fig. $\mathbf{n}^{\circ} \mathbf{6}$ ). Ce nouveau site n'était guère éloigné du quartier de cavalerie : les Frères avaient en effet repris les anciens locaux des Ursulines, qui s'étaient installées vers 1650 le long de la rue Saint-Ambroise, juste au sud des Visitandines. 


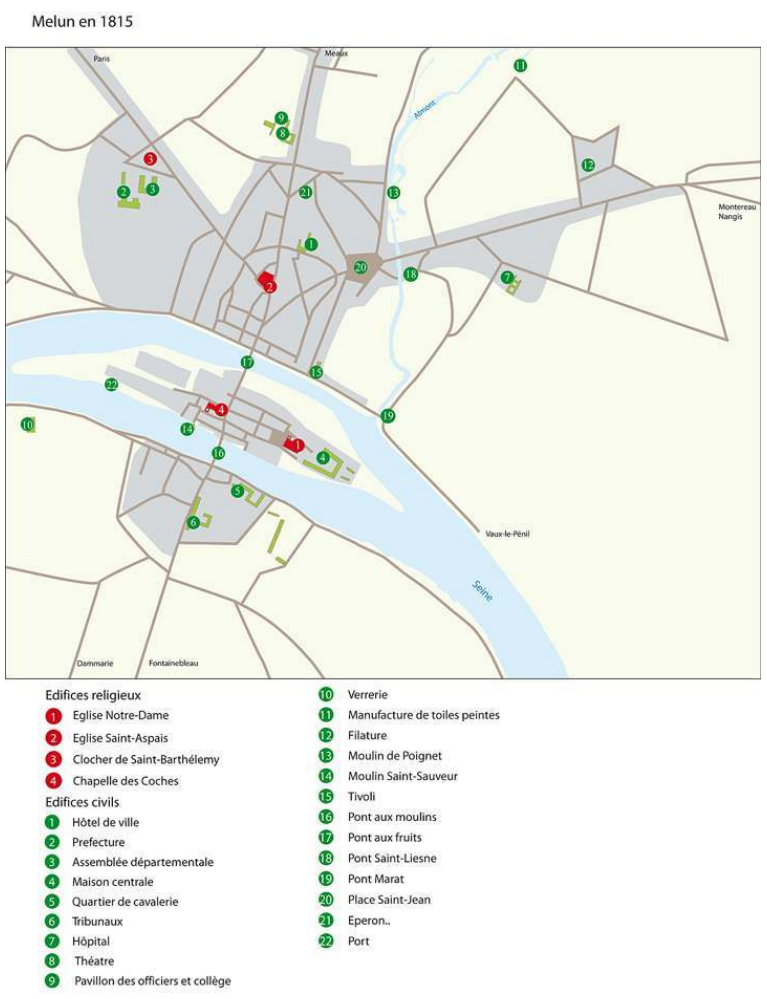

Melun en 1815. Bétored, Diane.

(c) Conseil régional d'île-de-France, service Patrimoines et Inventaire, 2007.

Le sort de ces deux anciens couvents allait d'ailleurs être réuni en 1816, lorsque l'armée céda à la Ville le "pavillon du quartier général », et reçut en échange le bâtiment du tribunal et des prisons - autrement dit le couvent des Frères. La caserne disposait dès lors d'un vaste emplacement, bordé au nord par la Seine et à l'ouest par la rue Saint-Ambroise (axe central de la ville, aboutissant au Pont-aux-moulins). Son effectif pouvait passer de 293 à 600 hommes. Sur un plan dressé en 1832 (fig. $\mathbf{n}^{\circ}$ 7), on voit clairement l'emprise des deux anciens couvents désormais réunis : au nord, le grand corps de logis (noté A sur le plan) correspond au bâtiment des Visitandines; au sud-ouest, un autre corps de logis (noté $\mathrm{K}$ ), au plan en $\mathrm{U}$, est quant à lui l'ancien bâtiment des Ursulines (alias des Frères de la Doctrine chrétienne). La comparaison avec un plan de $1772^{11}$ montre que ce bâtiment, qui abritait alors au rez-de-chaussée la cuisine et le réfectoire ainsi que la «salle de communauté ", a certainement conservé une partie de ses dispositions d'origine malgré d'indéniables remaniements. 
Figure 7

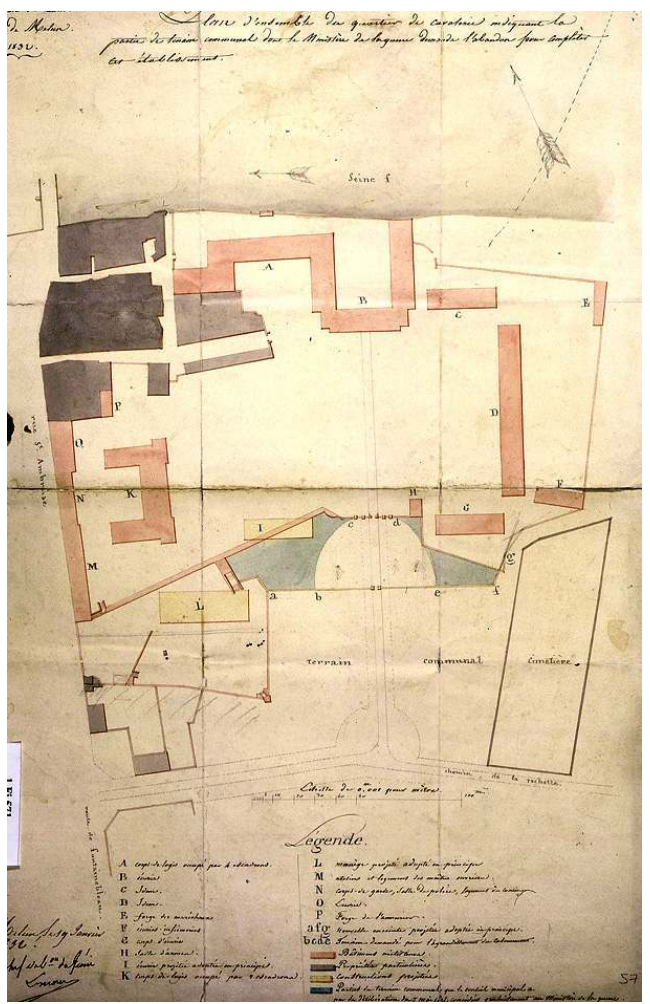

Plan du quartier de cavalerie en 1832. Archives municipales de Melun, 1 Fi 571. Repro. Asseline, Stéphane.

(c) Ville de Melun/Conseil régional d'île-de-France, service Patrimoines et Inventaire, 2002.

Tout au long du XIXe siècle, ce quartier de cavalerie ne cessa de s'étendre, jusqu'à devenir, selon l'historien melunais Gabriel Leroy, «une des plus vastes casernes de France $»^{12}$. Entre les deux anciens couvents se trouvait un petit lotissement de maisons particulières, appelé le " palais royal », que l'architecte Lesieur avait mis en œuvre sur le site de l'ancienne église paroissiale Saint-Ambroise (rachetée à l'occasion de la vente des biens nationaux). Ce lotissement fut englobé dans le quartier de cavalerie, et en grande partie détruit, en $1839^{13}$. Mais c'est surtout vers le sud-est que la caserne trouva à accroître son emprise, profitant du déplacement du cimetière Saint-Ambroise (1854). Elle put ainsi s'étendre, au sud, jusqu'à la rue de la Rochette et à l'est, jusqu'à la rue Dajot. Seuls les angles de ce vaste quadrilatère échappaient en partie à l'armée, avec la présence de l'usine des eaux (au nord-est) et de maisons particulières au sud-ouest et au nordouest (fig. $\mathbf{n}^{\circ} \mathbf{8}$ ). Dans ce mouvement d'extension, il n'y eut qu'un épisode de reflux, d'ailleurs mineur: l'armée dut accepter de céder une petite portion de son terrain au nord, le long de la Seine, pour que les Ponts et Chaussées puissent établir un chemin de halage en 1837. 
Figure 8

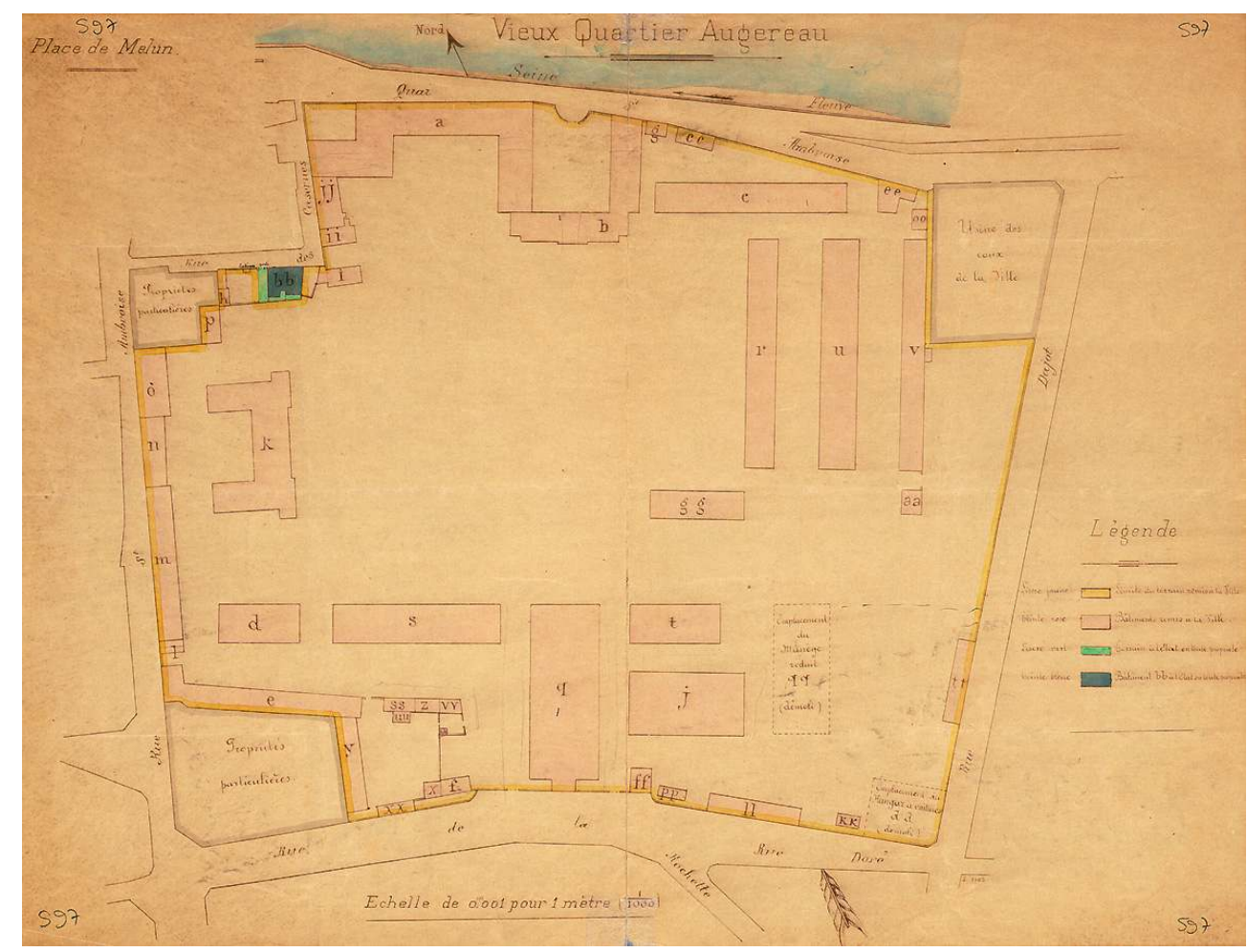

Plan du quartier de cavalerie en 1905. Archives municipales de Melun, 1 Fi 597. Repro. Archives de Melun.

(c) Ville de Melun/Conseil régional d'Île-de-France, service Patrimoines et Inventaire, 2004.

$\mathrm{Au}$ sein de cette emprise de plus en plus vaste, les ingénieurs du Génie élevèrent de nombreux bâtiments conformes aux directives du Ministère, notamment plusieurs corps d'écuries à l'est, et un manège couvert au sud (selon les plans de Picot, en 1850) (fig. $\mathbf{n}^{\circ}$ 9) . Ces divers aménagements entraînèrent la disparition graduelle des fortifications, dont il ne restait plus trace en 1900. Certes, ces fortifications avaient déjà été concédées aux Ursulines et aux Visitandines par le roi et, même si la Ville en avait récupéré la propriété à la désaffectation des deux couvents, elles n'avaient depuis longtemps plus de réelle fonction militaire. Néanmoins, leur tracé est resté jusqu'à nos jours présent dans le reste du tissu urbain, grâce à la transformation des anciens fossés en promenades et en voies de circulation (boulevard Gambetta, rue des Fossés, avenue Victor-Hugo, promenade Chamblain...). Ce n'est que sur l'emprise du quartier de cavalerie que les anciennes limites urbaines ont totalement disparu. On percevait encore dans les années 1840 l'existence d'un ancien éperon ${ }^{14}$, résultant du réaménagement de l'enceinte médiévale par l'ingénieur d'Henri IV Ambroise Bachot. Il fut par la suite nivelé, et ce n'est qu'à l'occasion d'une fouille archéologique exécutée en 1994 que l'on redécouvrit les vestiges de cette enceinte, sous l'école Cassagne ${ }^{15}$. 


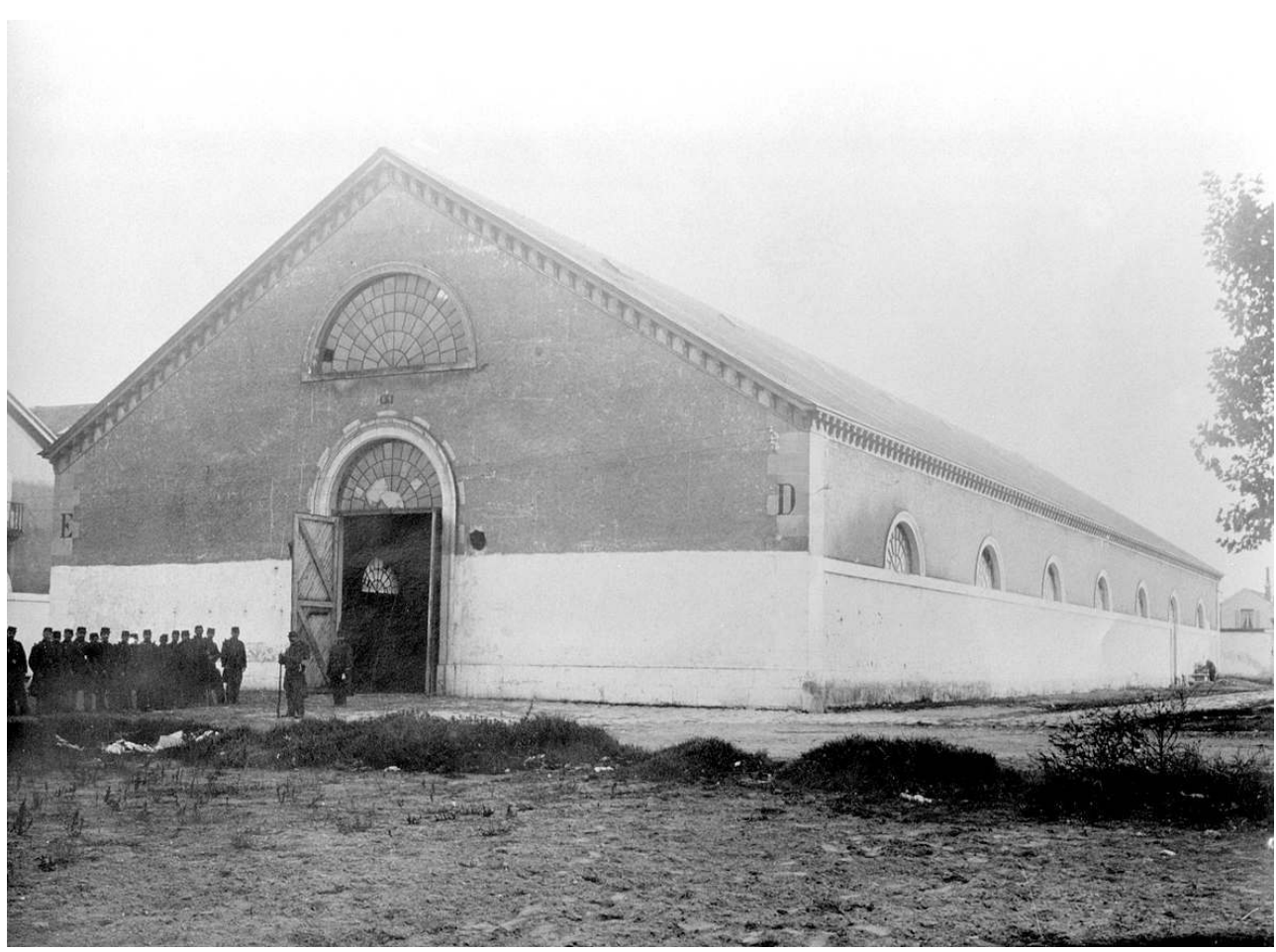

Le manège de cavalerie vers 1900. Collection particulière. Repro. Asseline, Stéphane.

(c) Conseil régional d'île-de-France, service Patrimoines et Inventaire, 2004.

11 S'il était de loin le plus important, le site du quartier de cavalerie Saint-Ambroise (rebaptisé « Augereau » en l'honneur du maréchal d'Empire) n'était pas le seul lieu dévolu à l'armée. Dans la première moitié du XIX ${ }^{e}$ siècle, elle acquit également deux anciennes usines : la verrerie du quai Hippolyte-Rossignol, transformée en manutention $(1822)^{16}$, et la manufacture de toiles peintes du faubourg Saint-Liesne, qui après une reconversion dans la production de sucre de betterave finit par devenir en 1841 une caserne d'infanterie (fig. $\mathbf{n}^{\circ}$ 10) $^{17}$. À ces deux établissements s'ajoutèrent un certain nombre de bâtiments liés à la présence de l'armée, comme celui de la Société des Lits militaires, blanchisserie industrielle située au 1, rue Dajot (à l'angle du quai Saint-Ambroise), qui travaillait pour le quartier de cavalerie voisin ${ }^{18}$. N'oublions pas non plus les terrains non bâtis dont la troupe avait l'usage pour son entraînement, tels que le champ de tir établi le long de l'Almont, près de la caserne d'infanterie ${ }^{19}$. Néanmoins, le quartier Augereau restait indéniablement le lieu le plus emblématique de la présence militaire à Melun, ne serait-ce que par ses dimensions inégalées. En outre, l'aménagement de la caserne SaintLiesne (ou caserne Breton) se révéla plus difficile qu'escompté, à tel point qu'au milieu du $\mathrm{XIX}^{\mathrm{e}}$ siècle, l'armée envisagea de s'en défaire : « la caserne Saint-Liesne est tellement mal située et tellement défectueuse sous tous les rapports que l'Inspecteur Général pense qu'il y a lieu de l'abandonner $»^{20}$. Elle se résolut finalement à la conserver, sans en être vraiment satisfaite : «Les bâtiments sont vieux, et de mauvaise construction. Néanmoins, conformément à l'avis du comité du 17 décembre 1861, le ministre de la Guerre a décidé le 16 janvier 1862 qu'il convenait de prolonger autant que possible l'existence de cette caserne $»^{21}$. D'importants travaux menés en 1867-1868, allant parfois jusqu'à la reconstruction complète, permirent cependant d'améliorer ces bâtiments qui restèrent donc en fonction jusqu'à la fin du XIX ${ }^{\mathrm{e}}$ siècle. En 1869, cette caserne avait une capacité 
d'accueil de 441 soldats, suffisante pour absorber les 400 hommes affectés en garnison à Melun par l'arrêt ministériel du 22 octobre 1857. Melun restait toutefois surtout dédiée à la cavalerie, la ville devant en 1859 abriter un régiment entier de la Garde, soit 1100 hommes et 810 chevaux.

Figure 10

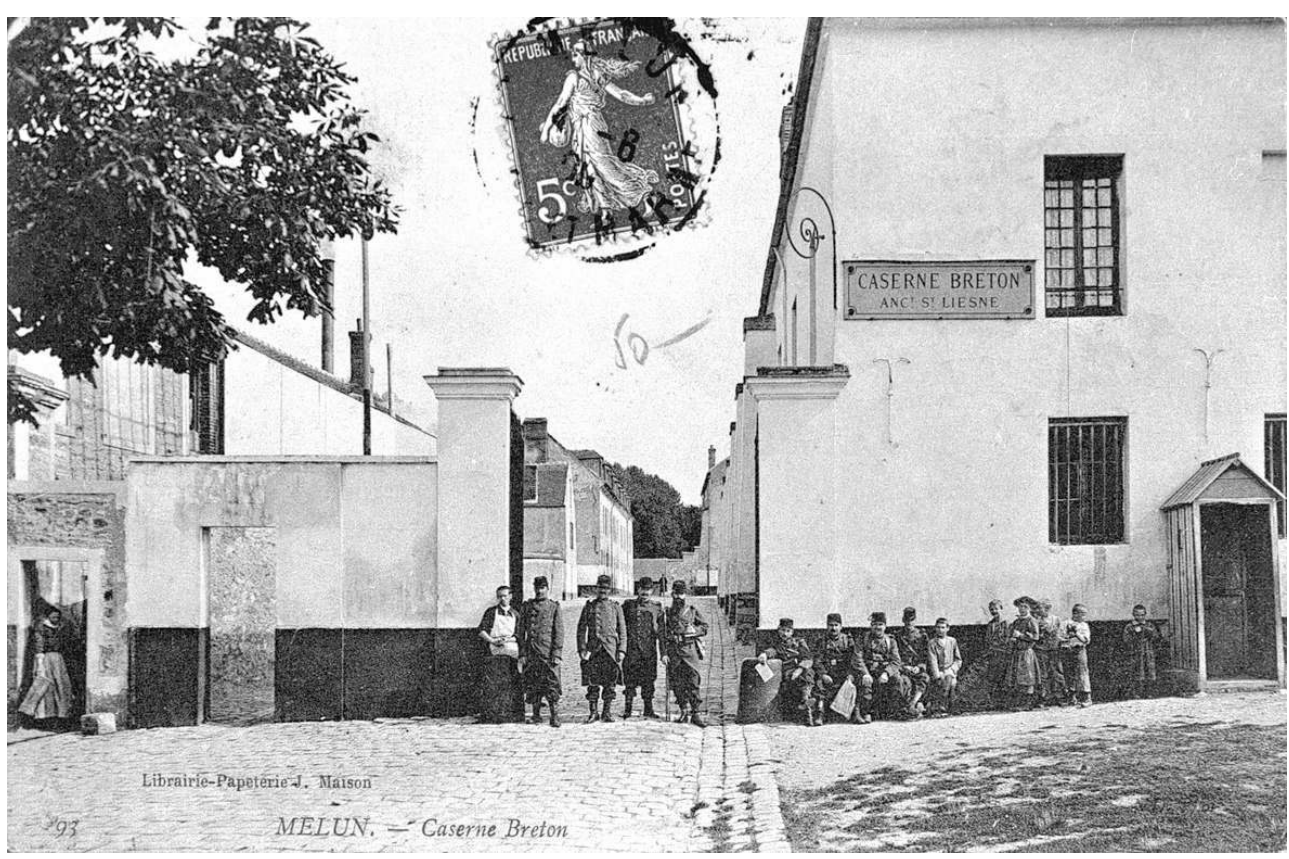

La caserne Breton, vers 1900. Carte postale conservée au musée de Melun, inv. 992.16.12. Repro. Asseline, Stéphane.

(c) Ville de Melun/Conseil régional d'Île-de-France, service Patrimoines et Inventaire, 2001.

C'est donc l'ensemble de la présence militaire à Melun qui fut remise en question par une épidémie de dysenterie, révélatrice de la vétusté du quartier de cavalerie Augereau, à partir de 1890. Malgré les mesures prises (remplacement de l'eau de Seine par celle de la source Saint-Liesne, changement des parquets, aspersions antiseptiques...), la maladie régnait encore de façon chronique en 1893, occasionnant plusieurs décès. L'Armée fit pression sur la Ville pour que celle-ci engage la construction de nouvelles casernes, menaçant de quitter Melun si l'ancien quartier restait en fonction. Le conseil municipal, soucieux de conserver sa garnison qui faisait vivre le commerce local, adopta le principe d'un transfert des troupes sur un autre site, au nord de l'agglomération (1894). En 1900, les bâtiments étaient construits mais ne purent être occupés immédiatement par la troupe, en raison d'un problème dans l'approvisionnement en eau. Ce n'est qu'en 1904 que le déménagement put enfin s'effectuer, le $18^{\mathrm{e}}$ Dragons et le $31^{\mathrm{e}}$ d'Infanterie quittant alors la caserne Saint-Ambroise pour gagner leurs nouveaux locaux (fig. $\left.\mathbf{n}^{\circ} \mathbf{1 1}\right)^{22}$. 


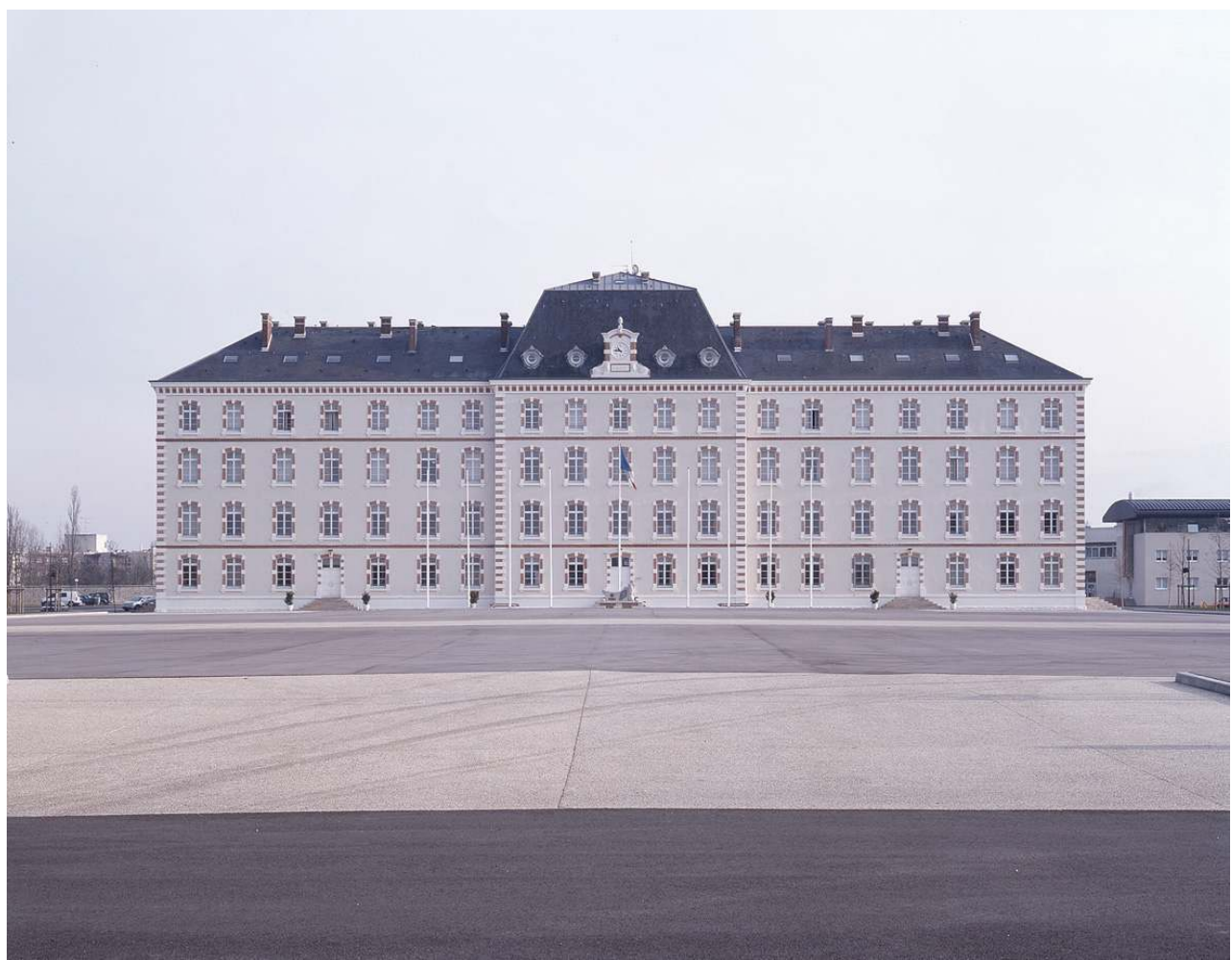

Les nouvelles casernes : le quartier Pajol. Photo Asseline, Stéphane.

(c) Conseil régional d'île-de-France, service Patrimoines et Inventaire, 2005.

Les considérations qui accompagnèrent la décision du transfert de la garnison sur le plateau nord montrent bien que les autorités municipales avaient pleinement conscience des conséquences qu'allait apporter ce changement pour l'urbanisme melunais :

«La ville de Melun, enserrée entre le remblai du chemin de fer et les coteaux qui la dominent, était paralysée dans son expansion ; la création du nouveau boulevard, la création du nouveau quartier en procurant des surfaces à bâtir, donneront à la partie nord de la ville un essor indiscutable. À la place de l'ancienne caserne s'élèvera aussi un nouveau quartier, conçu d'après les dispositions les plus modernes, qui ajoutera à la beauté de la ville et profitera aussi, on ne doit pas craindre de le dire, à la richesse de la partie sud encore plus que le casernement actuel $»^{23}$.

À la perspective d'embellissement de la ville s'ajoutent en effet des préoccupations économiques, qui sont clairement exprimées par le conseil municipal dans la même délibération :

« Nul doute qu'à la suite de la vente des terrains de la caserne Augereau, un nouveau quartier ne s'élève, fournissant à l'activité melunaise et à la population laborieuse de la ville de nouveaux éléments de prospérité. C'est du travail pour beaucoup d'années, pour les constructeurs de la ville, pour les ouvriers c'est un mouvement d'affaires inusité qui résulteront des travaux projetés. » 


\section{L'expansion urbaine}

Ainsi que l'analysaient les édiles, le départ de l'armée allait rendre à l'occupation urbaine toute une zone anciennement habitée, mais dont la trame viaire avait peu à peu été gommée par l'occupation militaire (fig. $\mathbf{n}^{\circ} \mathbf{1 2}$ ). Le quartier de cavalerie étant rétrocédé à la Ville, ce sont les autorités municipales qui prirent en main le dessin de ce nouveau quartier. L'implication de la municipalité dans la création du lotissement nous vaut une abondante documentation, conservée aux Archives municipales de Melun et aux Archives départementales de Seine-et-Marne. Le lotissement Saint-Ambroise est ainsi le mieux connu de tous ceux qui ont été mis en œuvre à Melun dans les années 1890-1914, époque de forte croissance urbaine : les autres opérations, menées par des promoteurs privés tels que Maurice Chamaillé, créateur du lotissement de la rue des Tilleuls, sont plus difficiles à appréhender. Le lotissement Saint-Ambroise se distingue en outre par la taille importante du terrain disponible, par sa situation en cœur de ville, et aussi par les préoccupations urbanistiques de ses concepteurs, qui eurent ici à cœur de prendre en compte la forme de la ville toute entière pour le siècle à venir.

Figure 12

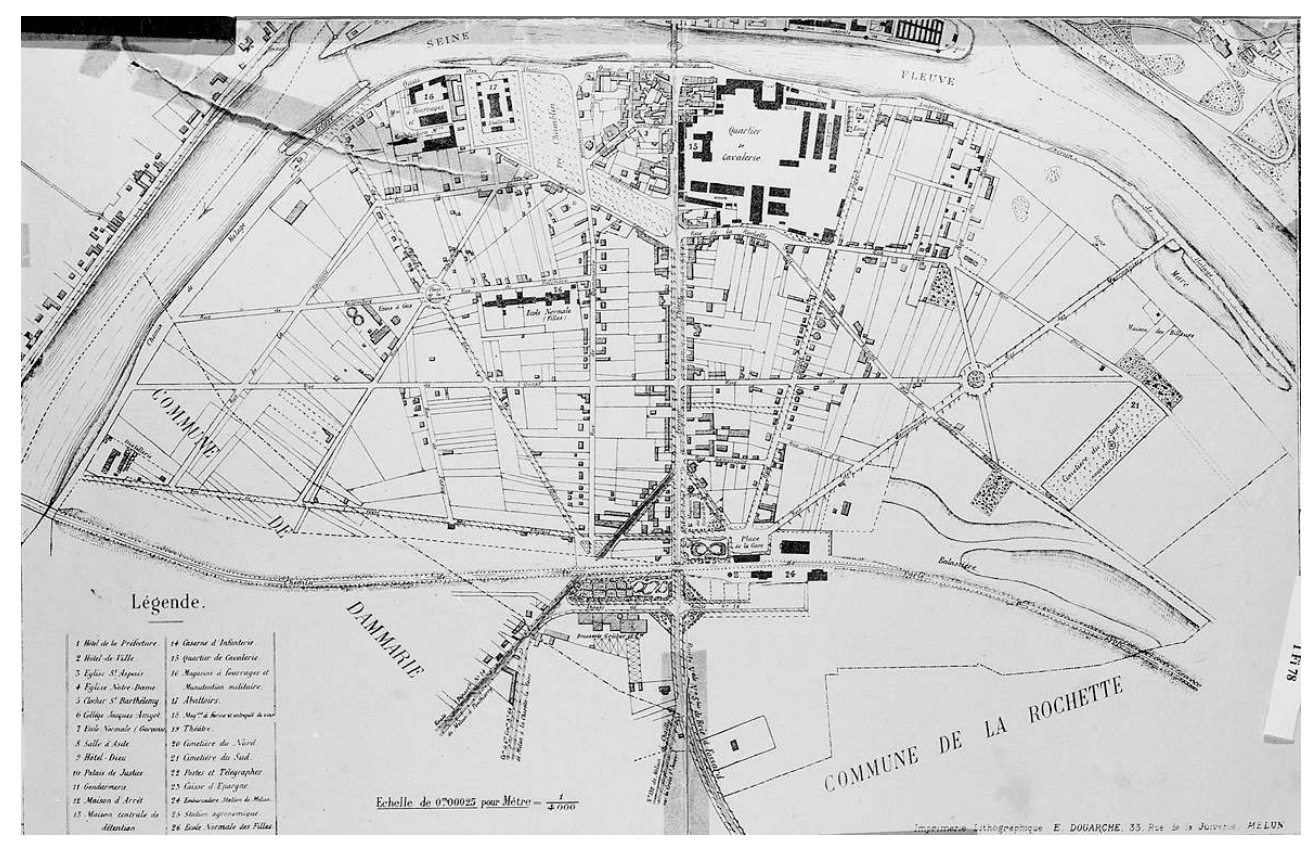

Plan de Melun-sud en 1889, par le géomètre A. Grosjean. Le quartier de cavalerie Augereau occupe une très vaste emprise au sein de la trame urbaine. Archives municipales de Melun, 1 Fi 78. Repro. Asseline, Stéphane.

(c) Ville de Melun/Conseil régional d'Île-de-France, service Patrimoines et Inventaire, 2002.

La municipalité a d'entrée de jeu pris la mesure de l'opportunité qui s'offrait à elle et commencé très tôt à réfléchir au réaménagement de l'ancien quartier de cavalerie. Dès 1899, en effet, à propos de la construction d'un immeuble neuf près de la Banque de France (qui faisait face au quartier de cavalerie, de l'autre côté de la rue Saint-Ambroise), le conseil municipal y vit « une amorce aux constructions qui se feront prochainement sur l'emplacement du quartier Augereau, quand ce quartier aura été rendu à la ville $»^{24}$. Un premier plan du lotissement était déjà établi en janvier 1905. Il fut toutefois l'objet 
d'abondantes discussions, d'abord dans le cadre de la commission créée à cet effet par le conseil municipal, puis au sein du conseil lui-même, avant d'aboutir à la version définitive du lotissement en août 1906.

Les débats qui ont tourné autour de ce plan sont très révélateurs des conceptions qui ont pu s'affronter autour de l'aménagement de ce vaste espace libre en pleine ville. Libre, car il fut entendu dès l'abord que l'armée ne comptait pas continuer à occuper le site, et que les bâtiments qu'elle avait édifiés à son usage seraient pour la majeure partie rasés ; seuls " certains bâtiments, de construction relativement récente, pourront être conservés et aménagés pour recevoir divers services municipaux $»^{25}$. Dans les faits, rares furent les édifices qui échappèrent à la pioche des démolisseurs. On conserva le manège de cavalerie, « que les Melunais verraient avec déplaisir disparaitre $»^{26}:$ il fut transformé en salle des fêtes, et on y remploya le portail d'honneur de la caserne (fig. $\left.\mathbf{n}^{\circ} \mathbf{1 3}\right)$. Deux écuries furent cédées à l'industriel Wilhelm Baumann qui les aménagea en ateliers pour son usine de stores $^{27}$. Les francs-maçons récupérèrent eux aussi un ancien bâtiment militaire, en bordure de la rue Dajot, pour en faire leur loge. On avait également envisagé de transformer en école un autre bâtiment de la caserne, mais ce projet fut abandonné et c'est un groupe scolaire entièrement neuf qui fut finalement édifié le long de la rue Armand-Cassagne.

Figure 13

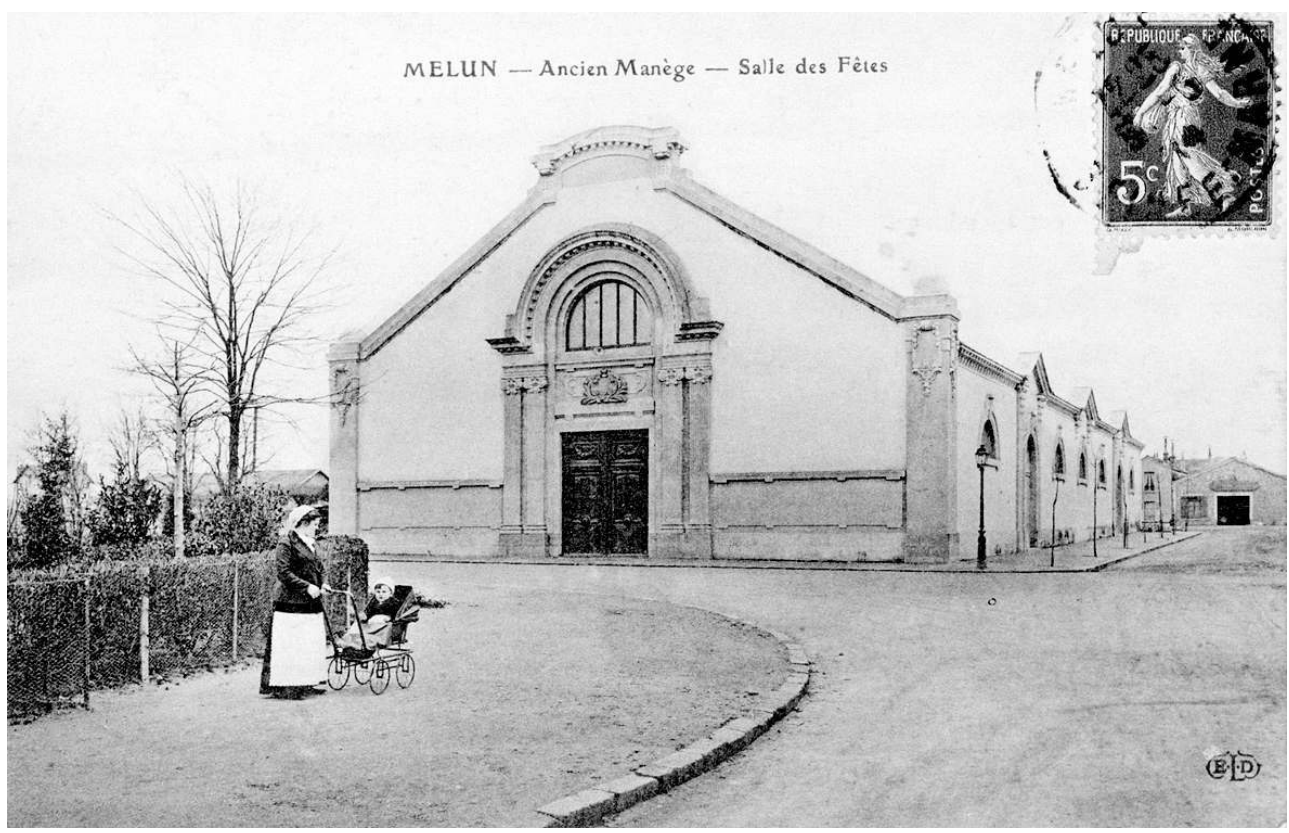

Le manège transformé en salle des fêtes. Carte postale, Médiathèque de Melun. Repro. Asseline, Stéphane.

(c) Ville de Melun/Conseil régional d'Île-de-France, service Patrimoines et Inventaire, 2002.

Le mot d'ordre général fut donc celui de la table rase, et l'on n'hésita pas à démolir la plus grande partie des constructions militaires. Disparurent ainsi les derniers vestiges de l'église Saint-Ambroise, ainsi que les bâtiments à arcades qui occupaient l'emplacement du couvent des Ursulines et de celui des Visitandines.

Comment organiser ce vaste espace de plus de 6 hectares ? Les contraintes étaient peu nombreuses. La Ville devait certes prévoir la construction d'une école maternelle et d'une 
«école primaire supérieure» de filles, qui viendrait remplacer l'établissement congréganiste du boulevard Gambetta (promis à fermeture dans le cadre de la loi de Séparation). Il lui fallait également envisager la conservation d'un bâtiment pour les besoins de l'armée, et la mise à disposition d'un terrain pour les bassins d'épuration de l'usine des eaux ${ }^{28}$. Restaient aussi les propriétés particulières que le quartier de cavalerie n'avait pas réussi à absorber, dans les angles du périmètre. Mais dans l'ensemble, la municipalité était entièrement libre de mettre en forme le quartier idéal du Melun moderne.

Deux principes guidèrent les réflexions. Pénétrée des principes d'hygiène alors en vogue, la Ville souhaitait aménager un lotissement aéré, avec un square ou tout au moins une esplanade plantée d'arbres. D'autre part, il fallait garantir une circulation harmonieuse non seulement au sein du lotissement, mais dans l'agglomération toute entière. Le tracé des voies nécessitait donc d'intégrer le quartier à venir dans une réflexion plus générale sur l'urbanisme melunais (fig. $\mathbf{n}^{\circ} \mathbf{1 4}$ ).

Figure 14

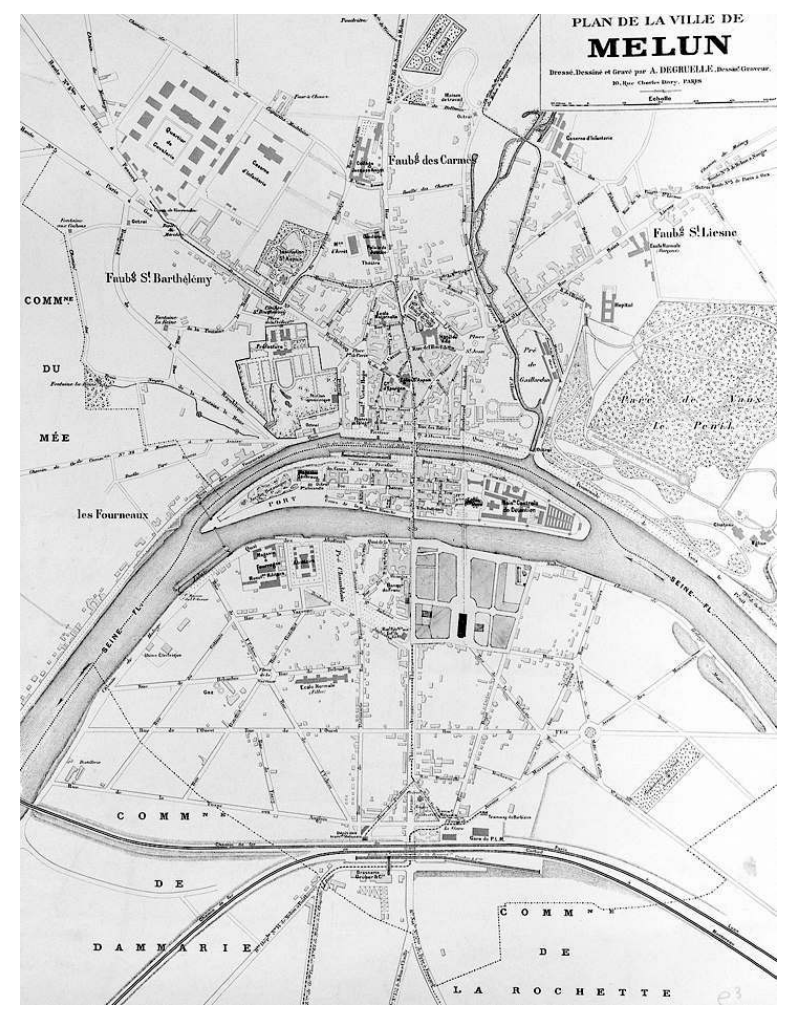

Plan de Melun vers 1905, par A. Degruelle. Le plan du lotissement Saint-Ambroise n'est pas encore arrêté, mais on a déjà prévu une large avenue vers la Seine, dans l'axe du boulevard Gambetta sur l'autre rive, en cas de doublement des ponts. Archives municipales de Melun, 1 Fi 391. Repro. Asseline, Stéphane.

(c) Ville de Melun/Conseil régional d'île-de-France, service Patrimoines et Inventaire, 2002.

21 Le rapporteur Bulot (par ailleurs architecte du département) souligne avec force le refus d'« un réseau de rues nouvelles isolées en elles-mêmes et sans lien avec la circulation générale ». Il fallait se montrer plus éclairé que les prédécesseurs. "Quand on jette un coup d'œil d'ensemble sur le plan général de la ville de Melun, on est frappé du manque de coordination des voies créées depuis une cinquantaine d'années entre l'ancienne ville, qui était à peu près limitée au sud par le pré Chamblain et la rue de la Rochette, et la voie 
du chemin de fer. Il semblerait qu'aucun plan d'ensemble n'ait été établi et que chacun ait planté une petite rue au gré de sa fantaisie.» Ces rues, à part la rue Dajot qui débouche sur la gare, «sont tracées pour ainsi dire comme les allées d'un jardin sans souci des besoins publics ${ }^{29}$. De fait, l'urbanisation de la plaine de la Varenne consécutive à l'arrivée du chemin de fer dans le sud de la commune (ligne PLM, en 1849) s'est réalisée autour d'axes relativement étroits, ne desservant qu'imparfaitement la gare ferroviaire (fig. $\mathbf{n}^{\circ}$ 15). La seule grande voie de communication est restée l'axe nord-sud qui traverse tout Melun, et qui correspond pour la rive gauche à l'avenue Thiers et à la rue SaintAmbroise. Une petite rue fut aménagée pour relier la gare à cette route principale, qui continua donc à concentrer toute la circulation.

\section{Figure 15}

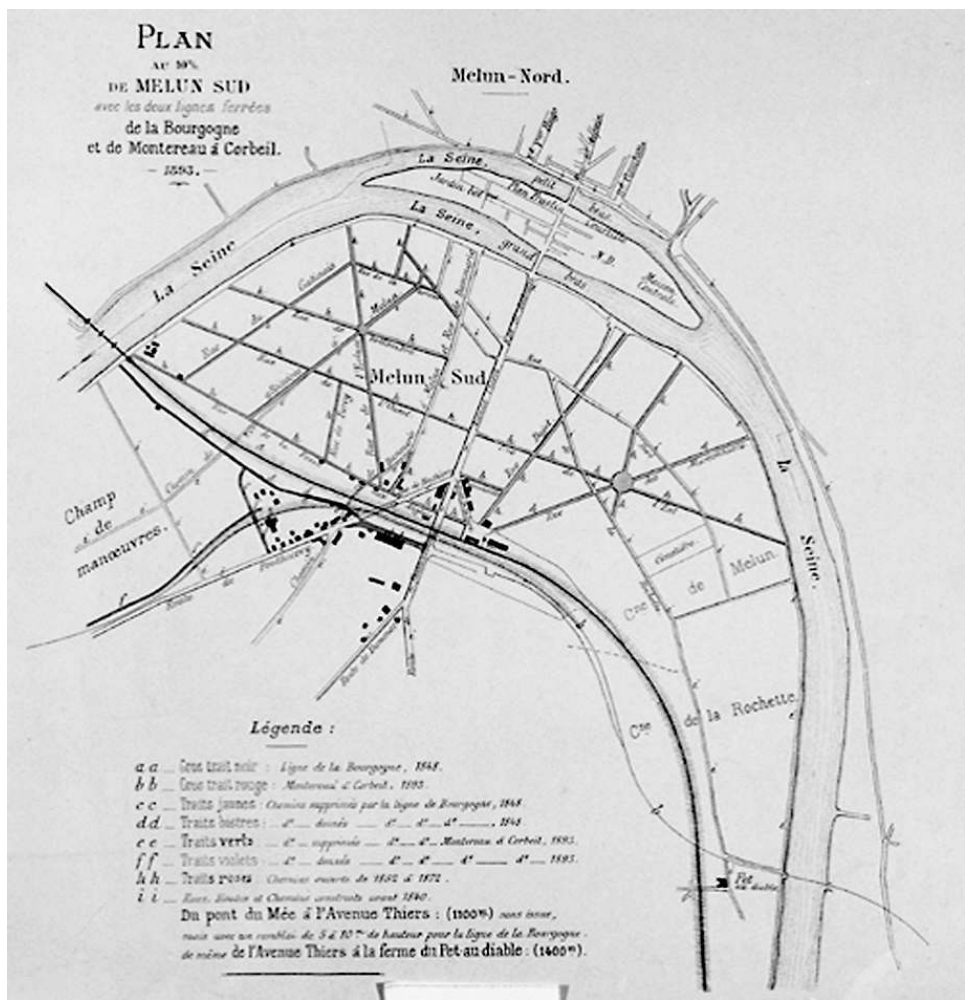

La trame viaire de Melun-sud, en 1893. La plupart des rues ont été ouvertes dans le $3^{\mathrm{e}}$ quart du XIXe siècle, en lien avec l'arrivée du chemin de fer. Archives municipales de Melun, 1 Fi 864. Repro. Asseline, Stéphane.

(c) Ville de Melun/Conseil régional d'île-de-France, service Patrimoines et Inventaire, 2002. 


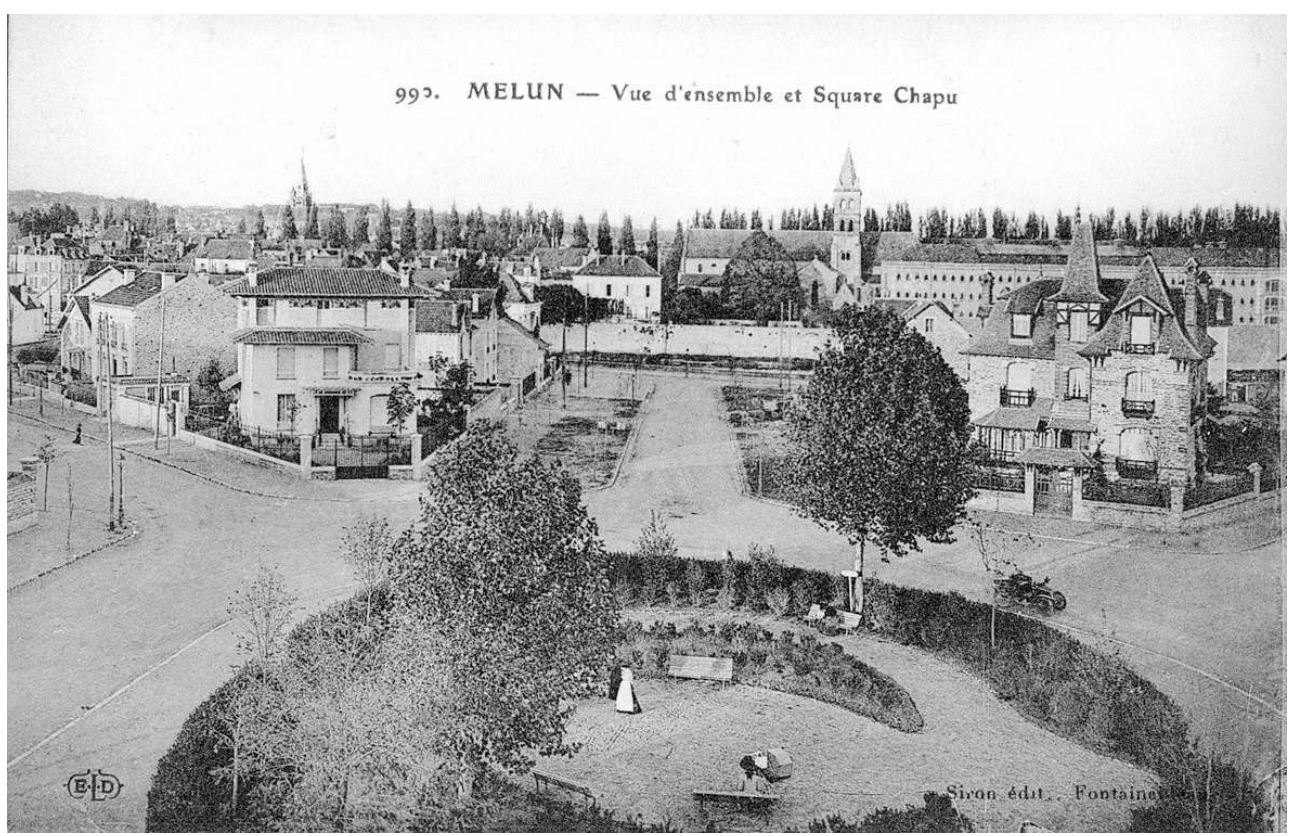

La partie nord du lotissement, avec sa large voie centrale ouvrant vers la Seine (état d'origine, avant la construction du pont Notre-Dame). Carte postale éditée par Siron, vers 1914. Musée de Melun, inv. 983.2.11. Repro. Asseline, Stéphane.

(c) Ville de Melun/Conseil régional d'île-de-France, service Patrimoines et Inventaire, 2001.

À l'époque de l'ouverture de ces nouvelles rues, l'ingénieur Dajot avait cependant préconisé le doublement de cet axe unique - ce qui impliquait la construction de nouveaux ponts sur la Seine, un peu en amont de ceux existant. Ce projet, s'il ne fut pas réalisé, était encore dans les esprits au tout début du $\mathrm{XX}^{\mathrm{e}}$ siècle. Il fut régulièrement évoqué au cours des travaux de la commission, et c'est dans l'attente de sa mise en œuvre qu'on résolut de percer une large voie centrale, face à l'église Notre-Dame. Cette voie pourrait un jour servir de débouché naturel aux deux ponts susceptibles de relier le boulevard Gambetta, sur la rive droite, et le nouveau quartier (voir fig. $\mathbf{n}^{\circ} 14$ ). En attendant, elle procurerait une agréable perspective sur la Seine (fig. $\mathbf{n}^{\circ} \mathbf{1 6}$ ). 


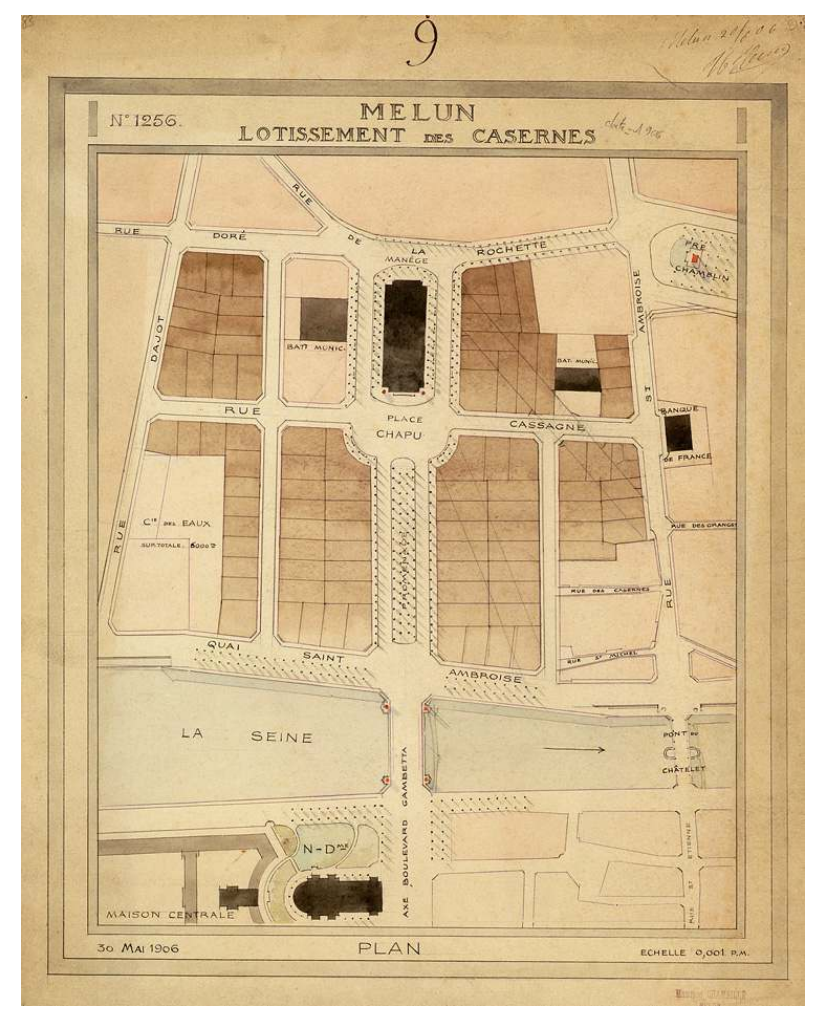

Projet pour le lotissement des casernes. Archives municipales de Melun, 1 Fi 2183. Repro. Archives de Melun.

(c) Ville de Melun/Conseil régional d'île-de-France, service Patrimoines et Inventaire, 2004.

Une fois ces principes établis et adoptés par l'ensemble de la commission, deux logiques s'affrontèrent quant au tracé de la voirie. La première, défendue notamment par le promoteur Chamaillé, était celle d'un tracé très géométrique : les axes se coupaient à angles droits, déterminant des lots rectangulaires (fig. $\mathbf{n}^{\circ} \mathbf{1 7}$ ). La seconde, qui finit par s'imposer, reposait sur des rues en diagonale facilitant le raccordement du Pont-de-fer à la rue Dajot, autrement dit la circulation entre le centre-ville et la gare (fig. $\left.\mathbf{n}^{\circ} \mathbf{1 8}\right)$. Pour son adversaire, «cette diagonale ne forme que des lots en trapèzes ou triangles évidemment impropres à la construction, en tous cas fortement dépréciés »; quant au square, il «constitue une perte sèche de terrain" dans ce quartier bourgeois «dont toutes les maisons sont clairsemées, entourées de jardins ». Chamaillé conclut sa diatribe en ces termes :

«Que notre ville consente quelques sacrifices pour trouer, désencombrer le quartier nord dont la densité est excessive, et qu'elle profite de l'économie de terrain résultant d'une renonciation aux espaces vides injustifiés, pour en faire bénéficier par des conditions spéciales de vente, des œuvres philanthropiques d'habitations ouvrières. Autant et plus que quiconque, je m'incline devant les nécessités de l'hygiène urbaine; exigeons par des règlements bien compris et intelligemment appliqués, des installations conformes aux exigences de la salubrité moderne. Que les rues bourgeoises soient astreintes à des zones non aedificandi pour en augmenter la largeur réelle sans perte de terrains, limitons la hauteur des constructions, imposons des surfaces minimum de cours, canalisons les égoûts, supprimons les formes fixes, imposons partout de l'eau et de la lumière, et nous aurons fait œuvre utile et profitable tout en dépensant peu. $»^{30}$ 
Figure 18

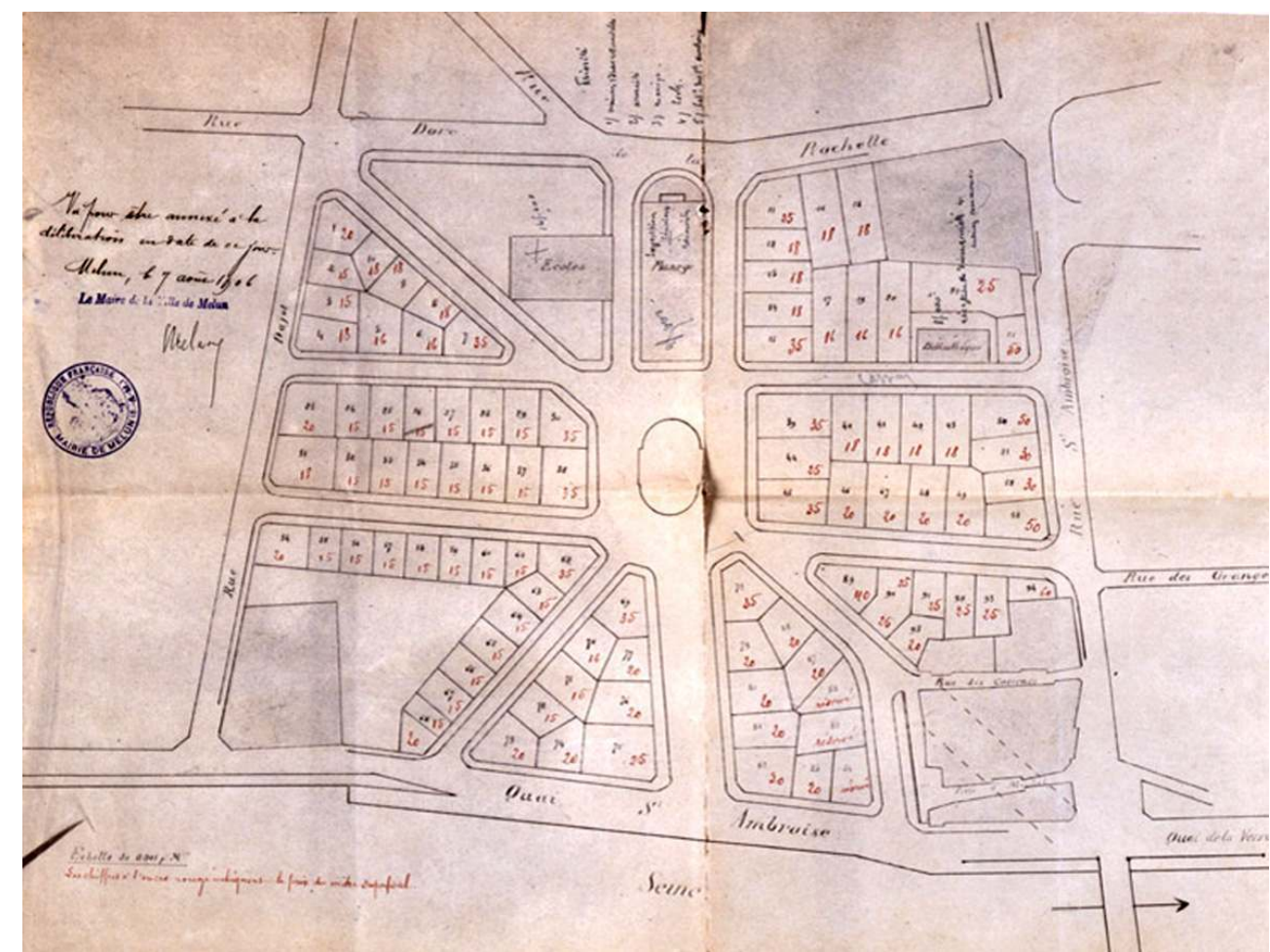

Plan du lotissement, 7 août 1906. Archives départementales de Seine-et-Marne, Op 273. Repro. Asseline, Stéphane.

(c) Archives départementales de Seine-et-Marne/Conseil régional d'île-de-France, service Patrimoines et Inventaire, 2006.

\section{Et il déplore :}

«Composée de fonctionnaires et de spécialistes distingués, la commission ne s'est pas, à mon avis, suffisamment arrêtée aux conséquences financières du projet qu'elle a adopté; j'aurais souhaité qu'elle comptât un plus grand nombre d'entrepreneurs, commerçants et propriétaires, en somme les plus intéressés par la question financière. »

Les positions vigoureuses de Chamaillé sont révélatrices d'une certaine conception de l'agencement de la ville, qui allait pouvoir se donner libre cours dans le projet d'aménagement de Champagne-sur-Seine, autour de l'usine Schneider. À Melun, ce sont toutefois les tenants d'un urbanisme moins pragmatique qui l'emportèrent, dotant le nouveau quartier de voies en diagonale convergeant autour d'un petit square central (fig. $\mathrm{n}^{\circ}$ 19). 


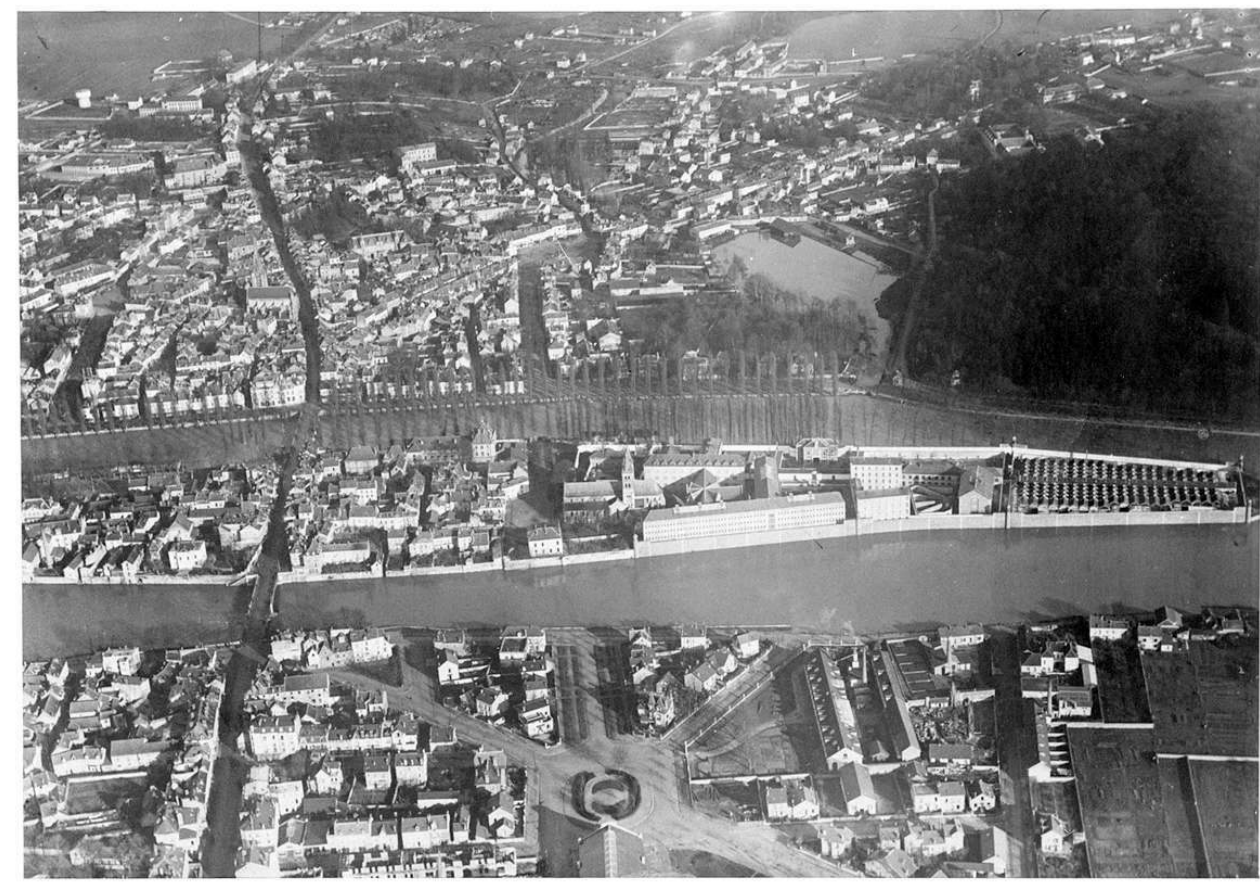

Vue aérienne du nouveau lotissement et de ses abords, vers 1915. Sur cette photographie apparaît nettement la possibilité d'un doublement de l'axe des ponts sur la Seine. Médiathèque de Melun, MM 1. Repro. Asseline, Stéphane.

(c) Ville de Melun/Conseil régional d'Île-de-France, service Patrimoines et Inventaire, 2002.

Comme l'avait prévu la municipalité, ce lotissement remporta un franc succès ${ }^{31}$. À la veille de la Première Guerre mondiale, la plupart des lots avaient été vendus et construits. Ainsi que l'avait voulu la Ville, le quartier reçut une population plutôt bourgeoise, surtout dans sa partie ouest. On y trouve de nombreuses villas qui, sans bénéficier de grands jardins, manifestent toutefois une intéressante recherche architecturale, à l'instar de la « villa del Sole» (d'ailleurs publiée dans la Construction moderne), bâtie en 1911 sur le mode italianisant par l'architecte Soubiran (fig. $\mathbf{n}^{\circ} \mathbf{2 0}$ ). Conformément au cahier des charges du lotissement, la rue Saint-Ambroise fut pour sa part bordée d'immeubles ayant au moins deux étages-carrés et une façade décorée. En revanche, la partie orientale du lotissement accueillit une architecture plus modeste, avec des pavillons construits par des entrepreneurs pour une population moins aisée ; c'est là aussi que se trouvaient l'usine des eaux et l'usine Baumann. 


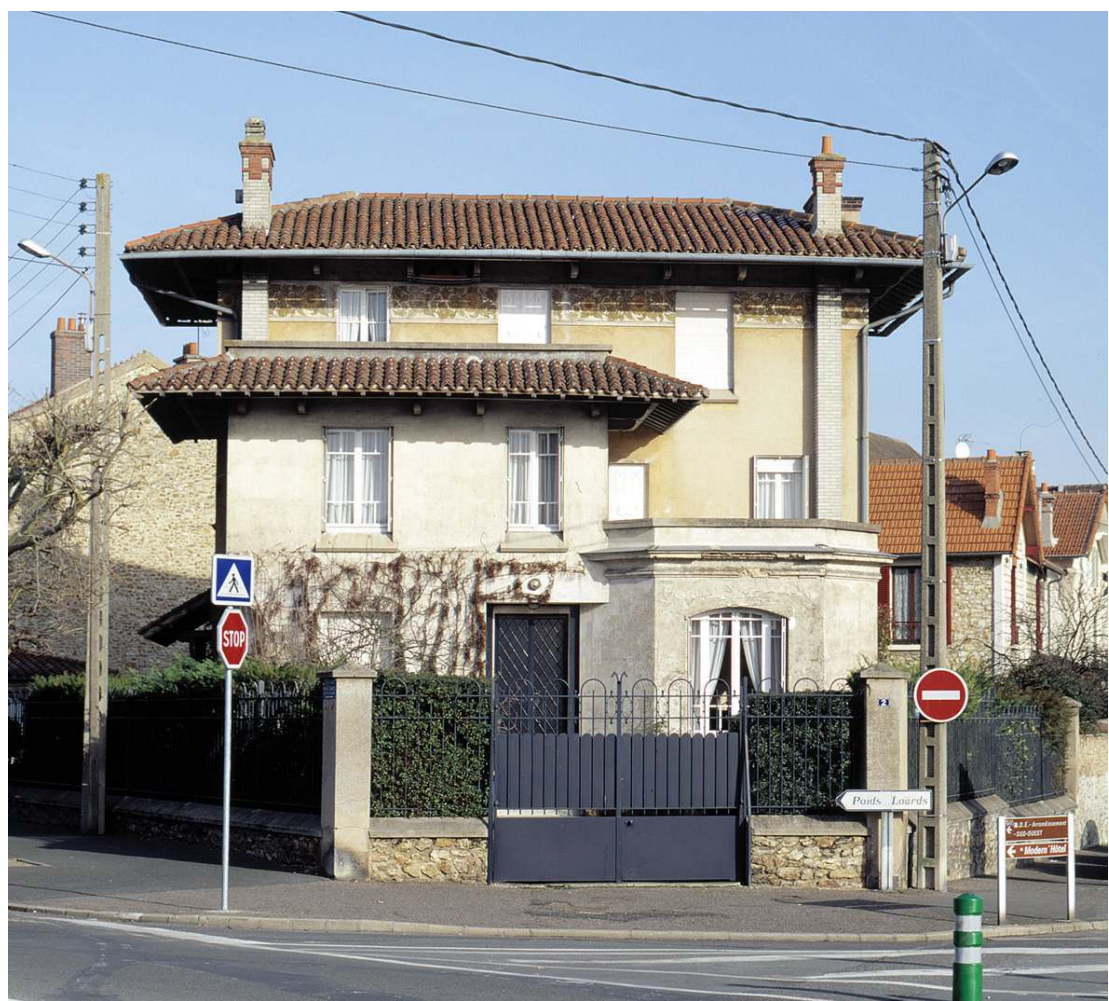

La villa « del Sole », 2 place Chapu. Photo Asseline, Stéphane.

(c) Conseil régional d'île-de-France, service Patrimoines et Inventaire, 2004.

Comme nous l'avons vu, la démarche urbanistique de la ville lors du transfert des casernes comprenait deux volets : d'un côté, aménager un secteur plein d'attraits sur le site de l'ancien quartier Augereau; de l'autre, développer l'agglomération vers le nord et vers l'ouest, autour des nouvelles casernes, avec la création du boulevard de la République (actuel boulevard Aristide-Briand), doublée d'un programme de construction d'égouts (voir fig. $\mathbf{n}^{\circ} \mathbf{1 4}$ ). Si l'on peut conclure au succès du premier volet, qu'en fut-il du second?

Sur le plateau et le long du coteau, l'urbanisation se fit plus lentement que dans le quartier Saint-Ambroise. Certes, des habitations furent élevées avant guerre le long de ce nouveau boulevard: trois d'entre elles, dues à l'architecte local Gérald de Faye, eurent même les honneurs d'une publication dans une revue d'architecture (fig. $\left.\mathbf{n}^{\circ} \mathbf{2 1}\right)^{32}$. Néanmoins, la plupart des maisons ne furent construites que dans les années 1920-1930. 


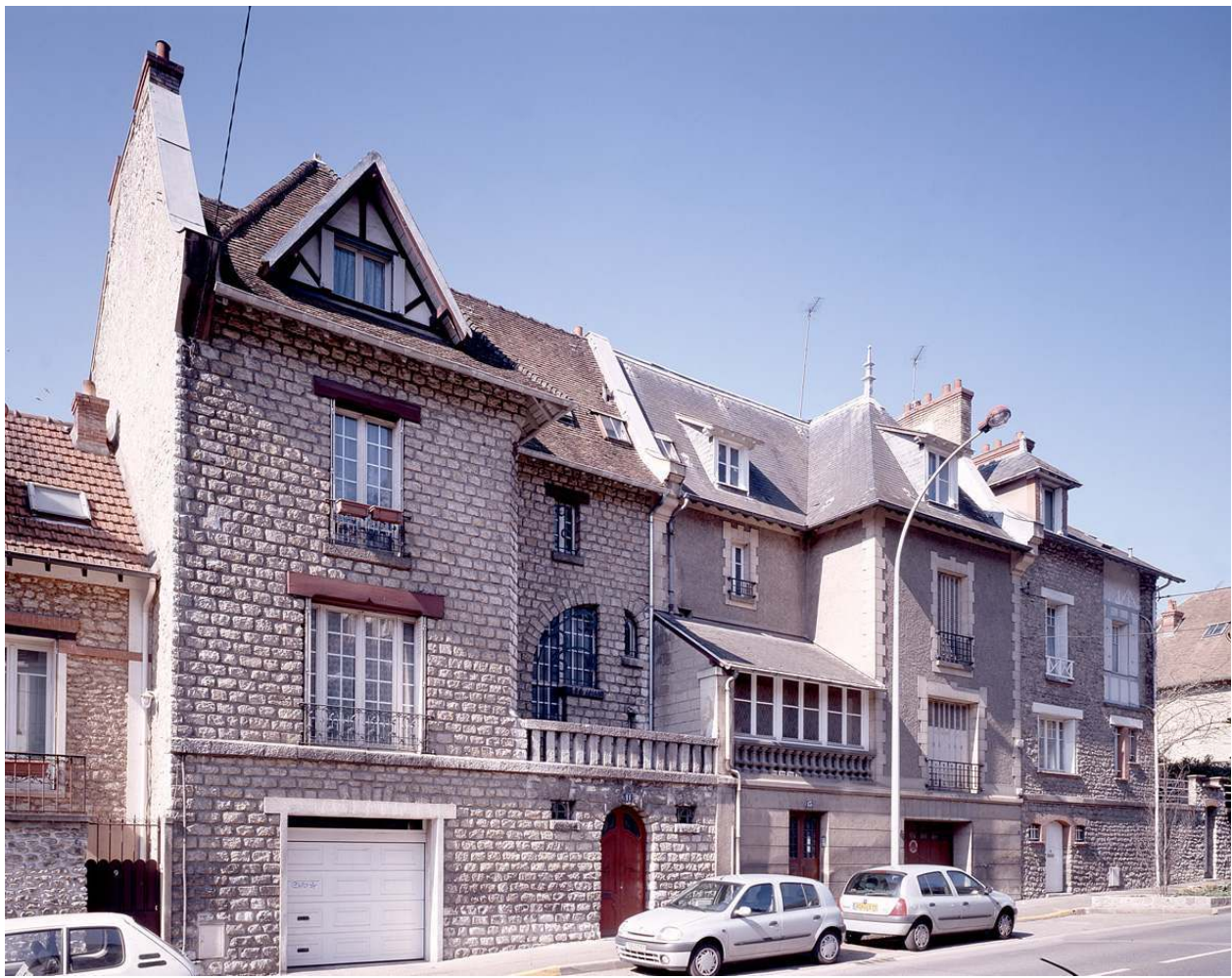

Le boulevard de la République : maisons élevées par l'architecte Gérald de Faye. Photo Asseline, Stéphane.

(c) Conseil régional d'île-de-France, service Patrimoines et Inventaire, 2004.

C'est donc surtout pendant l'Entre-deux-guerres que les nouvelles casernes jouèrent leur rôle de moteur de l'urbanisation du nord de Melun. La construction privée, le long du boulevard Aristide-Briand, fut relayée sur le plateau par une société d'HBM : le Foyer familial. Ce dernier mit en chantier un nouveau lotissement aux portes des casernes, le long de la route de Corbeil (fig. $\mathbf{n}^{\circ} \mathbf{2 2}$ ). Sur les 74 maisons individuelles ainsi programmées en 1931, 50 étaient spécifiquement destinées aux militaires ${ }^{33}$. 
Figure 22

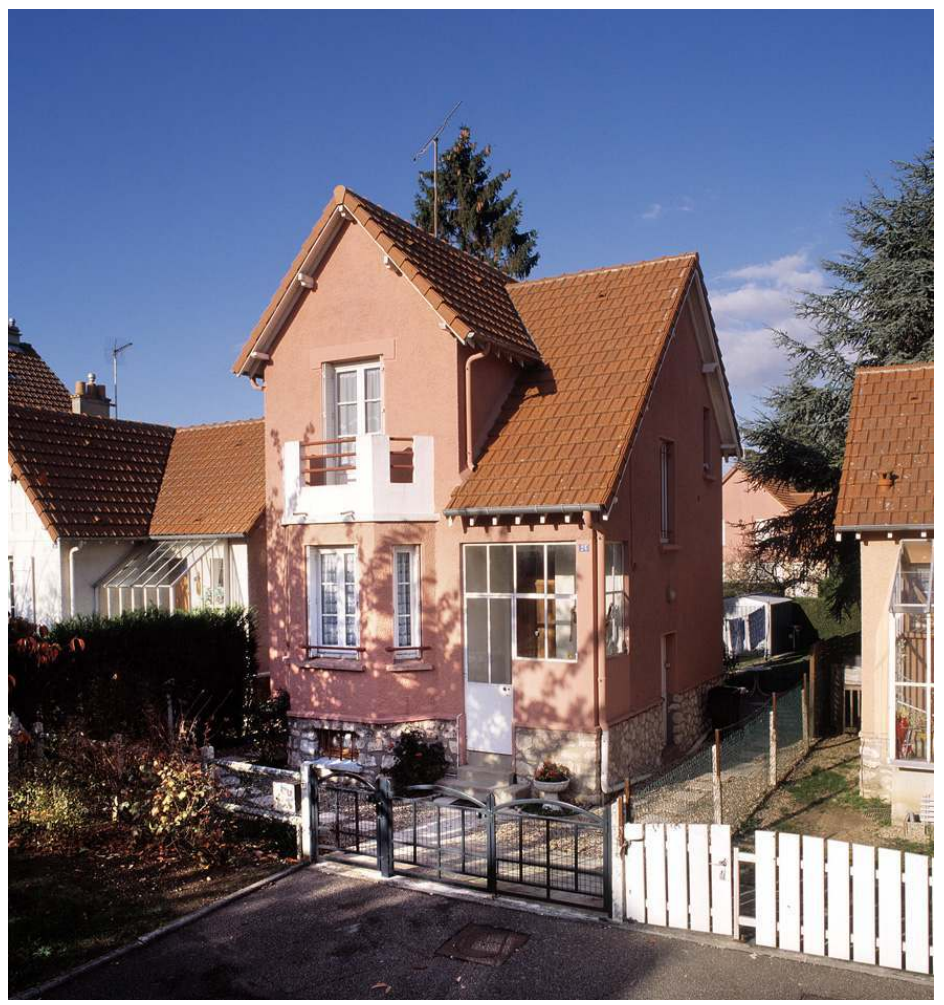

Une maison du lotissement du Foyer familial, route de Corbeil. Photo Asseline, Stéphane.

(c) Conseil régional d'île-de-France, service Patrimoines et Inventaire, 2002.

Comme on a pu l'observer dans bien d'autres villes, telles que Rennes, Noyon ou Saumur, la construction des casernes a donc bien entraîné une extension graduelle de l'emprise urbaine, ainsi que l'avaient escompté les autorités municipales ; mais ce phénomène a été assez lent. De plus, on ne retrouve pas à Melun la même qualité de construction que celle qu'on peut par exemple observer à Saumur, autour de l'École de cavalerie (voir les hôtels particuliers de la rue d'Alsace) ${ }^{34}$.

\section{Conclusion}

L'étude de Melun, qui n'est qu'une petite ville de garnison parmi tant d'autres, est cependant révélatrice de traits significatifs. D'une part, la ville s'est montrée assez attachée à la présence de l'armée pour consentir d'importants investissements, qui l'obligèrent à s'endetter lourdement. D'autre part, la municipalité a immédiatement perçu les retombées du déplacement des casernes en termes de développement urbain. À ce titre, ses prévisions ont été pleinement réalisées, et l'on peut saluer la pertinence des décisions prises dans les années 1900. Sur l'emplacement de l'ancien quartier de cavalerie s'est développé un lotissement bourgeois toujours apprécié aujourd'hui; par ailleurs, la grande esplanade qui avait été réservée en vue d'un éventuel doublement des ponts sur la Seine joue aujourd'hui ce rôle de grande voie de communication entre le boulevard Gambetta, l'avenue Thiers et la gare, suite à la mise en service du pont Notre-Dame (1975). Quant aux nouvelles casernes, elles ont entraîné l'urbanisation de la zone nordouest de la commune, comme l'avait programmé la municipalité en 1894. En définitive, la 
seule emprise militaire qui soit restée sans affectation est l'ancienne caserne Breton, dans le quartier Saint-Liesne. Un temps reconvertie en bureaux, cette petite caserne est aujourd'hui un lieu en friche, qui n'a pas influé sur le développement de la ville. L'ancienne manutention a quant à elle disparu dans les années 1970, pour laisser place à une gigantesque "Cité administrative » abritant divers services de l'État (rectorat, impôts...).

Les sites appartenant à l'armée sont donc aujourd'hui moins nombreux qu'au XIX ${ }^{\mathrm{e}}$ siècle, mais la présence militaire reste une donnée structurante de la ville. Les «nouvelles casernes " accueillent en effet depuis 1945 l'École des officiers de la gendarmerie nationale (E.O.G.N.), ainsi que le musée de la Gendarmerie. Contrairement au destin de bien des casernes, celle de Melun n'est donc pas menacée d'abandon par l'armée. Mais les questions qui étaient à l'ordre du jour au conseil municipal à Melun dans les années 1890-1905 sont en définitive celles qui se posent à bien des édiles aujourd'hui, lorsqu'il faut envisager le réaménagement de vastes superficies délaissées par les troupes... Situation souvent angoissante, mais qui peut se révéler « une chance historique de créer des projets urbains $»^{35}$.

\section{NOTES}

1. - PLOUVIER, Martine (dir.). Laon. Belle île en terre, (collection Cahiers du patrimoine, $\mathrm{n}^{\circ} 40$ ), tome 2. Amiens : A.G.I.R.-Pic., 1996, p. 218, p. 15 et p. 94.

2. - BONNET, Claire, DUCOURET, Bernard, MIGUEL, Sandra. Châlons-en-Champagne, (collection Images du patrimoine, $\mathrm{n}^{\circ}$ 246). Langres : Dominique Guéniot, 2007, p. 20.

3. - Cette étude a donné lieu à la publication d'un ouvrage : FÖRSTEL, Judith (avec la collaboration de Laurence de FINANCE, Yves GALLET, Delphine GILLARDIN, Fabrice HENRION, Diane LANELUC, Antoine LE BAS, Claire MABIRE LA CAILLE et Nicolas PIERROT; avant-propos de Dominique HERVIER), AUDUC, Arlette (dir.), ASSELINE, Stéphane (photographie). Melun. Une île, une ville. Patrimoine urbain de l'Antiquité à nos jours, (collection Cahiers du patrimoine, $\mathrm{n}^{\circ}$ 84). Paris : APPIF, 2006. Les fiches Mérimée et Palissy sont par ailleurs consultables sur les bases du ministère de la Culture et de la Communication : http://www.culture.gouv.fr/culture/inventai/ patrimoine/

4. - Service Historique de l'Armée de Terre

(S.H.A.T.), 1 VH 1117 à 1120.

5. - DALLEMAGNE, François. Les Casernes françaises. Paris : Picard, 1990, p. 60.

6. - Archives Municipales de Melun (désormais AMM), EE d 38, pièce 1.

7. - AN, $Q^{1} 1404$. Arrêt du conseil du 7 septembre 1737 ; contrat d'aliénation du château du 12 décembre 1737.

8. - CRON, Éric. Un complexe militaire en périphérie urbaine, dans GARRIGOU GRANDCHAMP, Pierre (dir.). Saumur, L'École de cavalerie. Histoire architecturale d'une cité du cheval militaire, (collection Cahiers du patrimoine, $\mathrm{n}^{\circ} 70$ ). Paris : Monum, Éditions du patrimoine, 2005, p. 267-280. 
9. - RÉAU, Marie-Thérèse. Fontenay-le-Comte, capitale du Bas-Poitou. Urbanisme et architecture, $\mathbf{X V}^{\mathrm{e}}-\mathrm{XIX}^{\mathrm{e}}$ siècle, (collection Cahiers du patrimoine, $\mathrm{n}^{\circ}$ 92). Nantes : Éditions 303, 2008, p. $177-178$.

10. - AN, $\mathrm{F}^{13} 322$.

11. - Médiathèque de Melun, GG 2.

12. - LEROY, Gabriel. Histoire de Melun depuis les temps les plus reculés jusqu'à nos jours, repr. en fac-similé de l'édition de 1887 (Melun : Drosne). Paris : Office d'édition et de diffusion du livre d'histoire, 1994, p. 25.

13. - S.H.A.T., 1 V 1118.

14. - Voir par exemple le plan des « Projets pour 1845 », S.H.A.T., 1 V 1118.

15. - Service archéologique municipal de Melun, dossier 77288128.

16. - Voir le dossier en ligne sur la base Mérimée : notice IA77000601.

17. - Sur cette ancienne manufacture, voir : FÖRSTEL, Judith. Des usines en ville. Évolution de la géographie industrielle de Melun, 1780-1914, Histoire urbaine, ${ }^{\circ} 26$, décembre 2009, p. 111-138 ; et le dossier en ligne sur la base Mérimée : notice IA77000444.

18. - À la suite du transfert de ce dernier, le site fut récupéré par l'usine pharmaceutique Vernin : voir le dossier sur cette usine dans la base Mérimée : notice IA77000568.

19. - AMM, $3 \mathrm{R} 2$.

20. - S.H.A.T., 1 V 1119.

21. - S.H.A.T., 1 V 1120.

22. - Voir le dossier en ligne sur la base Mérimée : notice IA77000445.

23. - Délibération du conseil municipal du 20 juin 1894.

24. - AMM, Registre de délibérations du conseil municipal n³4, p. 230 (séance du 11 mars 1899).

25. - Délibération du 23 septembre 1904. Pour le plan des lots de démolition (avec en gris, les bâtiments à conserver), 20 mars 1905 : AMM, 1 Fi 594.

26. - Délibération du 10 novembre 1906.

27. - AMM, 10 279. Lot vendu à Baumann par la Ville le 27 novembre 1907.

28. - Délibération du 9 mai 1905.

29. - Tous ces extraits du rapport de Bulot ont été lus devant le conseil municipal le 26 juin 1906.

30. - AMM, 10259.

31. - Voir GILLARDIN, Delphine. Le lotissement des casernes Augereau, dans Melun. Une île, une ville. Patrimoine urbain de l'Antiquité à nos jours, (collection Cahiers du patrimoine, $\mathrm{n}^{\circ} 84$ ). Paris : APPIF, 2006, p. 196-199.

32. - Voir le dossier en ligne sur la base Mérimée : notice IA77000541.

33. - Voir le dossier en ligne sur la base Mérimée : notice IA77000514.

34. - CRON, Éric. Un complexe militaire en périphérie urbaine, dans GARRIGOU GRANDCHAMP, Pierre (dir.). Saumur, L'École de cavalerie. Histoire architecturale d'une cité du cheval militaire, (collection Cahiers du patrimoine, $\mathrm{n}^{\circ} 70$ ). Paris : Monum, Éditions du patrimoine, 2005, p. 267-280.

35. - Voir les réflexions à ce propos d'Alain Villaret: http://www2.logement.gouv.fr/publi/ accesbat/coll98/098_100.PDF 


\section{RÉSUMÉS}

La présence de l'armée à Melun, préfecture du département de Seine-et-Marne, a eu une incidence non négligeable sur le développement de la ville au XIX ${ }^{\mathrm{e}}$ et au XX $\mathrm{XX}^{\mathrm{e}}$ siècle. En 1780, un quartier de cavalerie s'est en effet installé dans un ancien couvent, sur la rive gauche de la Seine, et a connu un développement ininterrompu jusqu'à la fin du XIX ${ }^{\mathrm{e}}$ siècle, avant d'être transféré au nord de la ville en 1905. L'espace ainsi dégagé en bordure du fleuve fut l'occasion pour la municipalité d'engager un important programme d'aménagement, tandis que la construction d'un vaste quartier militaire en périphérie de la ville entraînait l'urbanisation de nouveaux secteurs.

Melun, near Paris, is the main town in the departement of Seine-et-Marne and the presence of the military in the town had a considerable influence on its development throughout the nineteenth and twentieth centuries. In 1780 a cavalry regiment was installed at Melun in a disused convent on the south bank of the Seine. The military occupation of the neighbourhood grew until the end of the nineteenth century. Then, in 1905, the barracks were moved to the north of the town, leaving a space by the river where the local authorities developed a large housing estate. The construction of new military facilities on the outskirts of the town led to urban development in this neighbourhood too.

\section{INDEX}

Keywords : barracks, Baumann, Chamaillé, gendarmerie officers' school, manège, Mazin, Melun, military storehouse, riding academy, town-planning, Ursuline convent, Visitandines convent Mots-clés : Baumann, caserne, Chamaillé, couvent d'Ursulines, couvent de Visitandines, école des officiers de la gendarmerie nationale (EOGN), manège, manutention militaire, Mazin, Melun, urbanisme

\section{AUTEUR}

\section{JUDITH FÖRSTEL}

Conservateur du patrimoine, Service Patrimoines et Inventaire, conseil régional d'île-de-France judith.forstel@iledefrance.fr 